\title{
Effective Action of Vacuum: Semiclassical Approach
}

\author{
Ilya L. Shapiro \\ Departamento de Física - ICE, Universidade Federal de Juiz de Fora \\ Juiz de Fora, CEP: 36036-330, MG, Brazil 1
}

\begin{abstract}
.
We present brief, to great extent pedagogical review on renormalization in curved space-time and of some recent results on the derivation and better understanding of quantum corrections to the action of gravity. The paper is mainly devoted to the semiclassical approach, but we also discuss its importance for quantum gravity and string theory.
\end{abstract}

\section{Contents}

1 Introduction. Classical gravity and its applicability. 1

1.1 The Schwarzschild solution . . . . . . . . . . . . 2

1.2 Standard cosmological model . . . . . . . . . . . . . . 3

1.3 Planck units, fundamental scale and quantum effects . . . . . . . 4

2 The semiclassical approach: choice of the action 6

2.1 Classical action . . . . . . . . . . . . . 6

2.2 Note on inflation and the natural candidate for being the inflaton . . . 9

2.3 Spontaneous Symmetry Breaking and induced gravity . . . . . . . . 9

3 Effective Action and Renormalization $\quad 12$

3.1 Possible and impossible form of quantum corrections . . . . . . . . . 13

3.2 Calculational methods in curved spaces . . . . . . . . . . . . . . . . 14

3.3 One-loop divergences and renormalization . . . . . . . . . . . . . 16

$3.4 \overline{\mathrm{MS}}$ scheme - based renormalization group . . . . . . . . . . 18

4 Conformal anomaly and anomaly-induced EA 21

4.1 Derivation of conformal anomaly . . . . . . . . . . . . 22

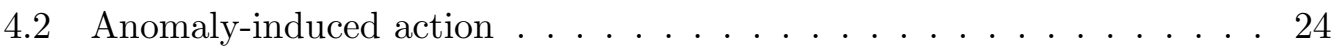

4.3 Light massive fields . . . . . . . . . . . . . . . . . . 26

5 Applications of the anomaly-induced action $\quad 29$

5.1 Vacuum states in the vicinity of a black hole . . . . . . . . . . . . . . . 29

5.2 Modified Starobinsky model. . . . . . . . . . . . . . . . 33

\footnotetext{
${ }^{1}$ Also at Tomsk State Pedagogical University, Russia. E-mail address: shapiro@fisica.ufjf.br
} 
6 Effective action for massive fields $\quad 36$

6.1 Decoupling theorem for gravity . . . . . . . . . . . 37

6.2 Massless limit in the massive theory . . . . . . . . . . . . . . . . . . . . . . . . . 38

6.2.1 Anomaly from the heat kernel solution. . . . . . . . . . . 39

6.2.2 Discontinuity of quantum corrections in the Proca theory . . . 40

7 Renormalization group for the cosmological constant 4

7.1 Once again on the physical renormalization group in curved space . . 41

7.2 Running cosmological constant . . . . . . . . . . . . 42

8 Do we need Quantum Gravity?

9 Conclusions

PACS: $04.62 .+\mathrm{v} \quad$ 11.10.Gh $11.15 .-\mathrm{q} \quad$ 11.30.-j

Keywords: Curved space, Effective Action, Renormalization Group, Conformal Anomaly.

\section{Introduction. Classical gravity and its applicability.}

One of the most important and well understood aspects of Quantum Gravity is the socalled semiclassical approach, where only matter fields are quantized, while metric is treated as a classical background. The main challenge in this area is deriving the quantum corrections to the classical action of gravity. Despite the form of these corrections is being, in general, unknown, there are strong hints that they may have numerous applications in cosmology and black hole physics. Moreover, depending on the role of these quantum contributions, one can or not justify the importance of other branches of Quantum Gravity, including string theory. In the present review paper, we describe a recent progress in calculating and better understanding quantum contributions to the effective action of gravity. Let us start by presenting the standard arguments for the necessity of quantum approach to gravity.

The modern gravitational physics is mainly based on General Relativity (GR). Correspondingly, a standard assumption is that the gravity action includes the Einstein-Hilbert term. The existence of the nonzero cosmological constant does not contradict any known principle and therefore it can be also included into the Lagrangian. So, the initial action has the form

$$
S_{E H}=-\frac{1}{16 \pi G} \int d^{4} x \sqrt{-g}(R+2 \Lambda) .
$$

The GR theory based on this action passed almost all known experimental and observational tests, however it is not free of unsolved problems. A serious one is, for instance, to explain the typical rotation curve observed in spiral galaxies. However, this problem can be solved by assuming the presence of an invisible Dark Matter - forming the halo in the region around the galaxy (see, e.g., the books [94, 88, 46, 81]). Alternatively, one can accept some modification in 
Newton's and Einstein's gravity laws [80, 19], although this option currently looks less favorable with respect to the full set of existing cosmological data. Another remarkable example of an unexplained gravitational phenomenon is the problem of Pioneer anomaly [2], but there are still chances to explain it in the framework of GR or some of its modifications at the classical level. This can be achieved either by introducing scalar fields in the braneworld scenario (see, e.g., [22]) or by introducing some ad hoc curvature dependence to the matter and gravity Lagrangians 23 . 2 .

All this shows that GR, despite its simplicity, beauty and efficiency, is not really a final word in the gravitational physics. On the top of that, GR has a serious conceptual problem related to the existence of singularities. These singularities emerge in the most important solutions such as spherically symmetric and cosmological ones. In the next two subsections we shortly consider these two solutions.

\subsection{The Schwarzschild solution}

The Schwarzschild solution corresponds to the spherical symmetry in the static mass distribution. In the simplest case of a point-like mass in the origin of the spherical coordinate system, the solution has the standard form

$$
d s^{2}=\left(1-\frac{2 G M}{r}\right) d t^{2}-\left(1-\frac{2 G M}{r}\right)^{-1} d r^{2}-r^{2} d \Omega
$$

where $d \Omega$ is the metric for the unit two-sphere. Let us notice that if performing an expansion of the above solution into the series in the parameter $1 / r$, one arrives at the relativistic corrections to the classical gravitational potential with relativistic corrections

$$
g_{00}=1+2 \varphi(r), \quad \varphi(r)=-\frac{G M}{r}+\frac{G^{2} M^{2}}{2 r^{2}}+\ldots .
$$

The Schwarzschild solution (2) contains two singularities: one at the gravitational radius $r_{g}=2 G M$ and second at the origin $r=0$. It is well-known that the first singularity is coordinatedependent (see e.g. [76, 127]). This singularity indicates the existence of a horizon, such that the light signals can not propagate from the interior of the black hole to its exterior. The same concerns, of course, the massive particles, which can never escape the interior of the black hole. However, the $r=r_{g}$ horizon looks as singularity only if it is observed from the safe distance. On the contrary, if the observer is changing his coordinate system to the Kruskal one,

$$
u=t-r-r_{g} \ln \left|\frac{r}{r_{g}}-1\right|, \quad v=t+r+r_{g} \ln \left|\frac{r}{r_{g}}-1\right|,
$$

the metric becomes regular at $r=r_{g}$,

$$
d s^{2}=-\left(1-\frac{r_{g}}{r}\right) d u d v+r^{2} d \Omega^{2} .
$$

The consistency of classical gravity is actually spoiled by the second singularity at the $r=0$ point. In the immediate vicinity of this singularity the curvature invariants grow infinitely and

\footnotetext{
${ }^{2}$ It is remarkable that modifications of similar form may show up due to the quantum corrections. This issue definitely deserves a detailed study.
} 
therefore it can not be cured by change of coordinates. Indeed, the Schwarzschild solution is valid only in the vacuum, and in reality one can not expect to meet point-like masses. The spherically symmetric solution inside the continuous matter does not have the $r=0$ singularity [76].

The fundamental significance of the $r=0$ singularity becomes clear after considering the phenomenon of the gravitational collapse, which is one of possible ways of the black hole formation (see e.g. [76, 56]). Qualitatively, the situation looks as follows: When the star (which has to be sufficiently massive) is losing its energy due to nuclear reactions and cools down, its size gets smaller, and the strength of the gravitational force on its surface increases. This process proceeds until the star becomes very small (a white dwarf or neutron star, depending on the initial state). Then, if the size of the star is large enough, the gravity force on the surface can become greater than certain limit, set up by the nuclear forces (Chandrasekhar or TolmanOppenheimer-Volkoff limits). In this case, the collapse of the star will continue and eventually its radius becomes smaller than its gravitational radius $r_{g}=2 G M$. For an external observer the star converts into a black hole.

The process of collapse will continue inside the black hole horizon (see, e.g, [56]). After all, if one assumes that the GR is valid at all scales, we arrive at the situation when the $r=0$ singularity becomes real. The matter energy density becomes infinite and so does the curvature. Our physical intuition tells that this is not a realistic situation and that some modifications are necessary in order to address this situation. The most natural option is perhaps to modify the action (1) in such a way which could prevent the formation of singularity at $r=0$.

\subsection{Standard cosmological model}

Another important solution of GR is the one for the homogeneous and isotropic metric, corresponding to the Friedmann-Lemaitre-Robertson-Walker (FLRW) solution. The most general form for the metric with these symmetries is

$$
d s^{2}=d t^{2}-a^{2}(t) \cdot\left(\frac{d r^{2}}{1-k r^{2}}+r^{2} d \Omega\right)
$$

where $r$ is the distance from some given point of the space, $a(t)$ is the conformal factor of the metric, and $k=0,1,-1$. The value of $k$ defines the curvature of the three-dimensional space section of the four-dimensional space-time manifold $M_{3+1}$.

The cosmological model is based on the Friedmann equation

$$
H^{2}=\left(\frac{\dot{a}}{a}\right)=\frac{8 \pi G}{3} \rho-\frac{k}{a^{2}}
$$

where $H=\frac{\dot{a}}{a}$ is a Hubble parameter and $\rho=\sum \rho_{k}$ is the total energy density, including contributions from different fluids, such as baryonic and dark matter, radiation, energy density of vacuum and maybe some additional unknown components. Furthermore, there are other equations, including the conservation law for the matter and vacuum components. In case of a single-fluid model we have

$$
\frac{d \rho}{\rho+P}=-\frac{3 d a}{a}
$$


For the matter-dominated epoch one can assume a zero pressure $P=0$. The solution corresponds, in the realistic situations, to the expanding Universe. This is in perfect agreement with the observational data, telling us that the present-day Universe is expanding according to the Hubble law. The most likely values of the relative vacuum energy densities are $\Omega_{v a c} \approx 0.75$ for the cosmological constant (Dark Energy), $\Omega_{M} \approx 0.25$ for the sum of dark and baryonic matter relative energy densities, $\Omega_{v a c} \approx 0.0001$ for the radiation relative energy density. The nowadays Hubble constant is $H_{0}=100 h_{0} K_{m ~ s e c}{ }^{-1} M p c^{-1}$, where $h_{0}=0.6 \pm 0.1$.

Due to the expansion of the Universe there was a radiation dominated epoch, when the cosmological constant was not relevant for the expansion. In this regime one can set the pressure $P=\rho / 3$ and then the solution has the form $a \propto t^{-1 / 2}$. In the limit $t \rightarrow 0$ this leads to the coordinate-independent singularity. Qualitatively the situation is quite similar to the one in the spherically symmetric solution. The classical solutions for the space-time manifold possess singularities and one would think whether modification of the gravitational equations are requested to cure this disease.

\subsection{Planck units, fundamental scale and quantum effects}

As we have just seen, the two most realistic solutions of GR end up with singularities. According to the dimensional arguments, in the regions close to singularities some quantum effects may be relevant. In a sufficiently close vicinity of the singularities one meets energy densities and curvature tensor components of a Planck order of magnitude.

The idea of the Planck units is the following. There are three fundamental constants in nature: the speed of light in vacuum $c$, the Planck constant $\hbar$ and the Newton constant $G$,

$$
c=3 \cdot 10^{10} \mathrm{~cm} / \mathrm{sec}, \quad \hbar=1.054 \cdot 10^{-27} \mathrm{erg} \cdot \mathrm{sec}, \quad G=6.67 \cdot 10^{-8} \frac{\mathrm{cm}^{3}}{\mathrm{sec}^{2} \mathrm{~g}} .
$$

It turns out that they can be used to construct the fundamental quantities with dimension of length $l_{P}$, time $t_{P}$ and mass $M_{P}$ :

$$
\begin{aligned}
l_{P} & =G^{1 / 2} \hbar^{1 / 2} c^{-3 / 2} \approx 1.4 \cdot 10^{-33} \mathrm{~cm} ; \\
t_{P} & =G^{1 / 2} \hbar^{1 / 2} c^{-5 / 2} \approx 0.7 \cdot 10^{-43} \mathrm{sec} \\
M_{P} & =G^{-1 / 2} \hbar^{1 / 2} c^{1 / 2} \approx 0.2 \cdot 10^{-5} \mathrm{~g} .
\end{aligned}
$$

These fundamental units can be interpreted in different ways. Let us start with the particle physics, where people use to put $c=\hbar=1$ and measure everything in $G e V$. Of course, in the everyday life this is not very nice, since you have to schedule the meeting with your friend "about $10^{27} \mathrm{GeV}^{-1}$ from now". It is actually the same thing, but "about 15 minutes from now" will be, perhaps, better appreciated. However, in a specific area of particle physics there are no factors like $10^{27}$ and the $G e V$ units are very useful. One can also measure the Newton constant in $G e V$, according to (10) we have $G=1 / M_{P}^{2}$. Of course, in these units $t_{P}=l_{P}=1 / M_{P}$. The numerical value is about $M_{P}=1.22 \times 10^{19} \mathrm{GeV}$.

One can suppose that the existence of fundamental units indicates the presence of some fundamental physics. Since the quantities (10) involve, simultaneously, $c, \hbar$ and $G$, we assume that the fundamental scale corresponds to some relativistic and simultaneously quantum, gravitational physics. One can think, for example, that due to the quantum effects, in the corresponding 
regions of the space-time manifold the gravitational theory has to be modified. The corrections may come from quantum matter, quantum gravity, supergravity, from the superstring theory, or from some yet unknown theory. In any case we have to assume the universal nature of these corrections. This means the gravitational action should be actually different from eq. (1) everywhere, not only in the vicinity of the singularities. And, since GR is a very successful theory, the first constraint is that, far from singularities, the effect of extra terms must be weak.

The dimensional analysis can not tell us an exact form of extra terms in the gravitational action. Also, we do not have certainty in what new kind of physics may be relevant for deriving these additional terms. Let us outline the following three main options:

i) Gravity and matter fields must be quantized at the Planck scale.

ii) Matter fields should be quantized, while gravity is an intrinsically classic interaction and hence should not be quantized at all. This looks like a reasonable option because, already in GR, gravity is different from other fields.

iii) Neither gravity nor matter fields should be quantized, both below and above the Planck scale. All we know as "fundamental interactions" emerge as effective low-energy effects of some unknown, really fundamental object, which must be, indeed, quantized at the Planck scale.

Let us start with a very brief description of the last paradigm iii) which, of course, corresponds to the (super)string theory [63]. In this theory the gravitational action is the low energy effective action of the background fields (metric, torsion, dilaton and their superpartners) of the really fundamental quantum object, that is a superstring. This effective action can be expanded into power series in the dimensional parameter $\alpha^{\prime}$. Einstein-Hilbert action corresponds to the lowest-order term of this expansion. Beyond this order one meets an infinite set of higher derivative terms. In any finite order in $\alpha^{\prime}$ these terms do suffer from a parametrization ambiguity. The origin of this ambiguity is that all fields, including the space-time metric, are nothing but external parameters for the quantum string. The reparametrization of these parameters does not spoil the consistency of the quantum theory of string [133. However, the physical effects which follow from the higher order corrections do depend essentially on such a reparametrization [78. On top of that, there are other ambiguities, for example those related to the compactification of extra dimensions. Finally, although the superstring theory is mathematically consistent, until now there is no clear way to extract falcifiable physical predictions out of it. Therefore, if we are interested in studying quantum corrections to General Relativity, it is worthwhile not to restrict our attention on the option iii), but also look somewhere else.

In the rest of our review we shall mainly deal with option ii) and just comment on the option $i$ ) now and, once again, in the special section 8. Of course, option $i$ ) looks more general, more fundamental and therefore more attractive. Furthermore, there is a standard argument in favor of quantizing gravity, related to a quantum mechanical inconsistency of the semiclassical approach ii) (see, e.g., [126] and references therein). These inconsistency, however, may be seen not as an argument in favor of quantizing gravity, but as a certain indication to the change of quantum mechanical laws, at least in the vicinity of singularities where the discrepancy mentioned above can be observed.

The main disadvantage of quantizing gravity is the known difficulty in formulating a consistent version of perturbative quantum field theory for the gravitational field. The attempts of quantizing gravity started long ago and at some point it became clear that the quantum theory based on general relativity is not renormalizable [74, 43]. Alternative theories with higher 
derivatives may be renormalizable [115] or even superrenormalizable [8] but they have unphysical components, called massive ghosts, in the spectrum and, at least if being treated as usual quantum field theories on flat background, this may lead to the violation of unitarity [115]. Indeed, there is a chance that the quantum corrections will make such theories unitary [118, 5], but the verification of this is, for a moment, beyond our possibilities [67].

Is it true that string theory is a real thing? Is it true that gravity should be quantized? We do not know the answers to these questions at the moment and it is not clear whether we will find them out soon. On the other hand, there are some certain and safe things and all of them concern the option ii) of our list. The success of the Standard Model of particle physics (SM) shows the correctness of the quantum field theory (QFT) description of the particles interactions. One of the most important aspects of QFT is the complicated vacuum structure which implies vacuum polarization, the possibility of particle creation and, in general, relevant effects of the virtual loops of matter fields. The vacuum quantum effect of matter fields can affect the gravitational action and, in principle, can change a situation with the fundamental problems of classical gravity which were discussed above. It is important to keep in mind that these quantum effects do not correspond to some qualitatively new physics which may exist or not. Much on the contrary, they represent a relatively well known physics, considered in a more complicated environment, that is in an external gravitational field.

Looking from this perspective, the most natural question is: - in which way vacuum quantum effects of matter fields, e.g., in the framework of SM or GUT's, do contribute to the gravitational action? The complete answer to the last question is unknown. The main purpose of this review is a survey of known corrections from quantum matter fields and their most important physical implications. At the end, we shall also discuss how the semiclassical results can, in principle, affect the necessity of a more complete quantum gravitational theory.

\section{The semiclassical approach: choice of the action}

The introduction to standard QFT in curved space can be found in the books [129, 64, 25, 31, 56, 83. In this and consequent sections we just review some fundamental aspects of the theory.

\subsection{Classical action}

The first step is to formulate quantum theory of matter on classical curved background, that means, at the first place, to define the classical actions for matter fields and for gravity. One can formulate these actions in infinitely many ways, so let us discuss only the simplest and most natural versions of the theory which provide the consistency at quantum level. Following this line, we impose the principles of locality and general covariance for both matter and gravity sectors. Furthermore, in order to preserve the fundamental features of the original flat-space theory, one has to require the symmetries (gauge invariance, at the first place) which take place in flat space-time, to hold for the more general theory in curved space-time. Even after that the number of possible terms in the action is unbounded, so we need some additional restriction. The natural requirement for the theory in curved space is renormalizability and, as we have already mentioned, simplicity. As we shall see in what follows, these two conditions 
can be satisfied even if we forbid new parameters with the inverse-mass dimension. This set of conditions enables one to construct the consistent quantum theory of matter fields on the classical gravitational background. Following the mentioned three principles (locality, covariance and restricted dimension), the form of the action is fixed except the values of some new parameters which remain arbitrary. The procedure which we have described above, leads to the so-called non-minimal actions.

Besides the nonminimal scheme for constructing the actions in curved space, described above, there is also a more simple, minimal one. According to it the partial derivatives $\partial_{\mu}$ are substituted by the covariant ones $\nabla_{\mu}$, the flat metric $\eta_{\mu \nu}$ by $g_{\mu \nu}$ and the volume element $d^{4} x$ by the covariant expression $d^{4} x \sqrt{-g}$. Let us compare the results of the two approaches. For the free scalar field the nonminimal generalization of the action is

$$
S_{\text {scal }}=\frac{1}{2} \int d^{4} x \sqrt{-g}\left\{g^{\mu \nu} \partial_{\mu} \varphi \partial_{\nu} \varphi+m^{2} \varphi^{2}+\xi \varphi^{2} R\right\}
$$

If compared to the flat-space theory, the action (11) involves a new dimensionless quantity $\xi$ which is called nonminimal parameter. The minimal version has $\xi=0$. It is easy to check that the $\xi$-dependent term is the unique one which is admitted in the scalar sector by the three principles imposed above. In the case of the multi-scalar theory $S\left[\varphi^{i}\right]$ the nonminimal term is $\int d^{4} x \sqrt{-g} \xi_{i j} \varphi^{i} \varphi^{j} R$. The generalization to the case of a charged scalar is obvious. The nonminimal term in the action (11) looks as a kind of modification of the massive term, despite there is a very essential difference between them. We shall discuss this difference in the subsection 2.3 .

It is very important that the mentioned three principles enable one to introduce just a finite number of matter-independent, purely vacuum terms. These terms represent a qualitatively new element compared to the flat-space theory. The most general action of vacuum, according to our principles, is as follows:

$$
S_{v a c}=S_{E H}+S_{H D}
$$

where $S_{E H}$ is the Einstein-Hilbert action with the cosmological constant (11) and

$$
S_{H D}=\int d^{4} x \sqrt{-g}\left\{a_{1} C^{2}+a_{2} E+a_{3} \square R+a_{4} R^{2}\right\}
$$

where $C^{2}=R_{\mu \nu \alpha \beta}^{2}-2 R_{\alpha \beta}^{2}+(1 / 3) R^{2}$ is the square of the Weyl tensor and $E=R_{\mu \nu \alpha \beta}^{2}-4 R_{\alpha \beta}^{2}+R^{2}$ is the integrand of the Gauss-Bonnet topological term. Let us remark that the presence of higher derivative terms is unavoidable is one wants have renormalizable theory. The same concerns the cosmological term, especially in case matter fields are massive.

The higher derivative terms in (12) are not quantum corrections, they should be introduced already at the classical level. The reason why they are not observed in the gravitational experiments is that the lower derivative Einstein-Hilbert term has the coefficient $1 / G=M_{P}^{2}$. Independent on whether we quantize gravity or not it is useful to use the language of Feynman diagrams. For example, the Newton law is the consequence of one-graviton exchange between the two masses. The higher derivative terms produce an additional particle similar to the graviton, but with the mass of the order $m_{2} \sim M_{P} / \sqrt{\left|a_{1}\right|}$ or $m_{0} \sim M_{P} / \sqrt{\left|a_{4}\right|}$, depending on the sector (spin-two or spin zero) of the propagator [116]. The exchange of these new particles 
produce modifications of the Newton law, but these corrections are small for the small values of transfered momenta, for the propagator can be presented, e.g., as

$$
\frac{1}{k^{2}+m_{2}^{2}}=\frac{1}{m_{2}^{2}}\left(1-\frac{k^{2}}{m_{2}^{2}}+\frac{k^{4}}{m_{2}^{4}}-\ldots\right) \text {. }
$$

It is obvious that, in order to have relevant impact of the higher derivative terms on the Newton law one needs the energy of graviton comparable to the Planck mass. Of course, the difference between the Planck scale and the present-day astrophysical scale is huge. As a consequence the higher derivative terms are irrelevant not only in the Solar System but also in most of the astrophysical objects, maybe except the black holes and sources of the gamma-ray bursts. In cosmology the higher derivative terms can be relevant only in the very early stage of the evolution of the Universe, e.g. in the Starobinsky inflation [114.

In order to complete the story, let us notice that the covariant, gauge invariant and local actions for fermion and vector fields are minimal, because no non-minimal terms are alrebraically possible. For the spinors we have

$$
S_{\text {fermion }}=i \int d^{4} x \sqrt{-g}\left(\bar{\psi} \gamma^{\alpha} \nabla_{\alpha} \psi-i m \bar{\psi} \psi\right)
$$

where $\gamma^{\mu}$ and $\nabla_{\mu}$ are gamma-matrix and covariant derivative of the spinor in curved space-time. For the gauge fields the action is

$$
S_{v e c t o r}=-\frac{1}{4} \int d^{4} x \sqrt{-g} G_{\mu \nu}^{a} G^{a \mu \nu}, \quad \text { where } \quad G_{\mu \nu}^{a}=\partial_{\mu} A_{\nu}^{a}-\partial_{\nu} A_{\mu}^{a}+g f^{a b c} A_{\mu}^{b} A_{\nu}^{c} .
$$

The interactions between the matter fields (gauge, Yukawa and self-scalar ones) are defined through the minimal procedure, because there are no nonminimal extensions compatible with the principles declared above. Finally, the vacuum action has universal form (12), independent on the choice of the fields and their interactions.

The last remark concerns a very important feature of the massless version of the matter fields and vacuum actions formulated above. In the scalar case (11) the value $\xi=1 / 6$ corresponds to the local conformal symmetry, that means the equations of motion of the theory do not change under the transformation

$$
g_{\mu \nu} \rightarrow g_{\mu \nu}^{\prime}=g_{\mu \nu} e^{2 \sigma(x)} \quad \varphi \rightarrow \varphi^{\prime}=\varphi e^{-\sigma(x)} .
$$

For spinor and vector fields in curved space the conformal transformations have the form

$$
A_{\mu} \rightarrow A_{\mu}^{\prime}=A_{\mu} \quad \psi \rightarrow \psi^{\prime}=\varphi e^{-3 \sigma(x) / 2}
$$

The local conformal symmetry of the free actions for these fields does not require anything but the masslessness. The form of the Noether identity corresponding to the symmetry under the local conformal transformation is

$$
\left[2 g_{\mu \nu} \frac{\delta}{\delta g_{\mu \nu}}+\sum_{i} k_{i} \Phi_{i} \frac{\delta}{\delta \Phi_{i}}\right] S\left(g_{\mu \nu}, \Phi_{i}\right)=0,
$$

where $k_{i}=(-1,-3 / 2,0)$ are the conformal weights of the matter fields $\Phi_{i}=\left(\varphi, \psi, A_{\mu}\right)$. 
In the vacuum action (12), only the higher derivative terms may satisfy the Noether identity (18). The condition of conformal symmetry for the action (13) has the form $a_{4}=0$. Let us notice that only the Weyl term $\int \sqrt{-g} C^{2}$ is a real conformal invariant, while the topological $\int \sqrt{-g} E$ and surface $\int \sqrt{-g} \square R$ terms in the vacuum action do change under the metric transformation (17). However, since they do not contribute to the equations of motion, the conformal Noether identity is satisfied for these terms.

It is a custom (or, better say, tradition) to consider the higher derivative terms (13) as producing the energy-momentum tensor $T_{\mu}^{\nu}$ of vacuum. Then the Noether identity (18) can be seen as the condition of a zero trace, $T_{\mu}^{\mu}=0$. In the consequent sections we shall explore the important role of the violation of this condition at the quantum level. This violation is behind the most important applications of QFT in curved space-time, such as Hawking radiation [70, 41] and Starobinsky model of inflation [114].

\subsection{Note on inflation and the natural candidate for being the inflaton}

Inflation is one of the most natural applications of Quantum Field Theory in curved spacetime, because it occurs at very high energies, where the quantum effects may be indeed relevant. An increasing interest to inflation emerged after the paper by Guth [65], where he noticed that the period of extremely fast expansion of the Universe possesses the following two properties: a) Solves numerous problems of the standard cosmological scenario [94, 75] such as the ones of flatness, horizon, monopole etc; b) Can be results of the Spontaneous Symmetry Breaking in the Standard Model of particle physics. In other words, the naturality of inflation is directly related to the natural origin of inflaton, which is nothing else but the Higgs field in the original proposal [65]. Let us notice, by passing, that the original proposal for the SM-based inflation may be rescued from the known difficulties [77] by the non-minimal generalization of the Higgs

field, that is by adding the $\xi R H^{\dagger} H$-term to the Higgs potential [24]. Needless to say this step is also the most natural one in view of the critical importance of a nonminimal scalar-curvature coupling for the renormalizability of the theory. If the non-minimally interacted to gravity Higgs field really satisfies all tests of phenomenological cosmology, it can be seen as the first candidate to be inflaton!

Another possibility to have natural inflation is related to the account of the vacuum quantum effects, that is in the framework of original Starobinsky model [114] or its modified version [52, 108, 95]. We shall discuss the backgrounds of these models in section 5 .

\subsection{Spontaneous Symmetry Breaking and induced gravity}

Before starting the consideration of the loop effects, it is worthwhile to spend some time and discuss one important aspect of the tree-level QFT in curved space-time. It is a classical issue in the sense it does not depend on the loop corrections. On the other hand, we shall observe here one typically quantum property, that is the presence of non-localities. Even more important is that we shall meet here a very important aspect of QFT in curved space, that is the possibility to induce the gravitational action. Let us remember that the QFT models of our main concern are the Minimal Standard Model of particle physics and also its extensions and generalizations, such as GUT's (Grand Unification Theories). The definition of vacuum is a very important element of these theories and it is usually performed through the Spontaneous 
Symmetry Breaking (SSB) and the Higgs mechanism. So, let us check, following [62], what is the impact of an external gravitational field here.

It is well known that the SSB leads to the induced cosmological constant [132] and in general to the induced gravity [104] (see [1] for a general review of induced gravity). Here we shall arrive at the induced action of gravity in a most natural way and also observe the emergence of the nonminimal terms. We associate the scalar (11) with the Higgs field. Consider the classical potential

$$
V(\varphi)=-\mu_{0}^{2} \varphi^{*} \varphi+\lambda\left(\varphi^{*} \varphi\right)^{2}-\xi R \varphi^{*} \varphi .
$$

The VEV for the scalar field is defined as a solution of the equation of motion

$$
-\square v+\mu_{0}^{2} v+\xi R v-2 \lambda v^{3}=0 .
$$

If the interaction between scalar and metric is minimal $\xi=0$, the SSB is standard and simple, because the vacuum solution of the last equation is constant

$$
v_{0}^{2}=\frac{\mu_{0}^{2}}{2 \lambda}
$$

where we have introduced a special notation $v_{0}$ for the case of a minimal interaction, in order to distinguish it from the solution $v$ of the general equation (20). The consistency of the QFT in curved space requires the non-minimal interaction such that $\xi \neq 0$. Then, for a general case of a non-constant scalar curvature one meets, instead of Eq. (21), another solution $v(x) \neq$ const. The derivatives of $v$ can not be ignored and, therefore, the solution for the VEV can not be obtained in a closed and simple form.

For the slowly varying curvature one can use the eq. (21) as the zero-order approximation. The solution of (20) can be found in the form of the power series in $\xi$,

$$
v(x)=v_{0}+v_{1}(x)+v_{2}(x)+\ldots .
$$

For the first order term $v_{1}(x)$ there is equation

$$
-\square v_{1}+\mu^{2} v_{1}+\xi R v_{0}-6 \lambda v_{0}^{2} v_{1}=0 .
$$

The solution has the form

$$
v_{1}=\frac{\xi v_{0}}{\square-\mu^{2}+6 \lambda v_{0}^{2}} R=\frac{\xi v_{0}}{\square+4 \lambda v_{0}^{2}} R,
$$

where we used (21). In a similar way, we find

$$
v_{2}=\frac{\xi^{2} v_{0}}{\square+4 \lambda v_{0}^{2}} R \frac{1}{\square+4 \lambda v_{0}^{2}} R-\frac{6 \lambda \xi^{2} v_{0}^{3}}{\square+4 \lambda v_{0}^{2}}\left(\frac{1}{\square+4 \lambda v_{0}^{2}} R\right)^{2},
$$

One can continue the expansion of $v$ to any desirable order, deriving $v_{3}, v_{4}$ etc.

Some observations are in order. Different from the usual SSB case, here the VEV of the scalar field is not a constant. Instead, it varies due to the variable curvature. Of course, this variation is completely negligible for the particle physics due to the extremely small value of 
curvature compared to any other dimensional quantity such as, e.g., $v_{0}$. Hence the impact of the gravitational interaction on the particle physics applications is irrelevant.

In the gravitational applications, however, the effect of SSB and nonlocalities is nontrivial. If one replace the SSB solution $v(x)$ back into the the action of the scalar field, the following result for the induced low-energy action of vacuum will follow:

$$
S_{i n d}=\int d^{4} x \sqrt{-g}\left\{g^{\mu \nu} \partial_{\mu} v \partial_{\nu} v+\left(\mu_{0}^{2}+\xi R\right) v^{2}-\lambda v^{4}\right\}
$$

In the lowest order of power expansion (23) the VEV of the scalar is $v \approx v_{0}$ and we meet the custom form of the induced Einstein and cosmological constant terms. The induced values of the Newton and cosmological constants correspond to

$$
\frac{1}{16 \pi G_{\text {ind }}}=-\xi v_{0}^{2}, \quad \frac{\Lambda_{\text {ind }}}{8 \pi G_{\text {ind }}}=-\mu_{0}^{2} v_{0}^{2}+\lambda v_{0}^{4}=-\lambda v_{0}^{4} .
$$

As we see, at the $v_{0}$ level, the low-energy induced action of gravity, due to the SSB, has the same form as the classical vacuum Einstein-Hilbert action with the cosmological constant. The difference between the two actions is that the constants $G$ and $\Lambda$ of the vacuum action (11) are independent parameters while the induced quantities (27) depend on the VEV and $\xi$. Other symmetries breaking may result in additional contributions to the induced action of gravity. In the simplest formulation, the induced quantities (27) have to be summed up with the vacuum quantities, that are independent parameters of the action (11) 3 . The observed quantities are the sums

$$
\frac{1}{G_{o b s}}=\frac{1}{G}+\frac{1}{G_{i n d}}, \quad \frac{\Lambda_{o b s}}{G_{o b s}}=\frac{\Lambda}{G}+\frac{\Lambda_{i n d}}{G_{i n d}} .
$$

It is interesting that both vacuum and induced quantities become subjects of renormalization at the quantum level.

If one compares the magnitudes of the vacuum and observed quantities, it is easy to notice that the effect of $G_{i n d}$ is quite small. For instance, consider $G_{i n d}$ in the framework of the Minimal Standard Model (MSM) or GUT. In case of MSM, $v_{0}$ is about $250 \mathrm{GeV}$ and therefore the induced quantity of $\left(G_{i n d}\right)^{-1}$ is about 32 orders of magnitude smaller than the observed one $\left(G_{o b s}\right)^{-1}$. The same difference is about 5 orders of magnitude in the GUT case. On the contrary, the induced value of the cosmological constant is enormous compared to the observed quantity, leading to the famous, important and mysterious cosmological constant problem. The induced cosmological term is supposed to almost cancel with its vacuum counterpart. The precision of the required cancelation is 55 orders of magnitude in the framework of the SM and even much more than that in GUTs and other generalizations of the SM. The nowaday physics can not explain the origin of this fine-tuning and this is one of the most difficult conceptual problems. One can find an extensive discussion of this problem in [128] and also, from the point of view of renormalization theory, in [110].

Beyond the lowest order one has to take into account the next terms in the expansion (22) and thus to account for the space and time dependence of the curvature scalar. It is remarkable

\footnotetext{
${ }^{3}$ In the more extreme version of the theory [1] the initial gravitational action is absent and all gravity is induced. This is indeed a very interesting and fruitful idea, but it goes beyond the purpose of the present review.
} 
that, along with the conventional cosmological constant and Einstein-Hilbert term, here we meet also an infinite series of non-local additional expressions due to non-localities in (22). For example, in the second order, by performing an expansion in the powers of the curvature tensor, we obtain much more complicated form of the induced gravity action

$$
\begin{array}{r}
S_{\text {ind }}=\int d^{4} x \sqrt{-g}\left\{-v_{1} \square v_{1}+\mu^{2}\left(v_{0}^{2}+2 v_{0} v_{1}+2 v_{0} v_{2}+v_{1}^{2}\right)\right. \\
\left.-\lambda\left(v_{0}^{4}+4 v_{0}^{3} v_{1}+4 v_{0}^{3} v_{2}+6 v_{0}^{2} v_{1}^{2}\right)+\xi R\left(v_{0}^{2}+2 v_{0} v_{1}\right)\right\}+\mathcal{O}\left(R^{3}\right) .
\end{array}
$$

Now, using the equation (23), after a small algebra we arrive at the following form of the action of induced gravity

$$
\begin{aligned}
S_{i n d} & =\int d^{4} x \sqrt{-g}\left\{\lambda v_{0}^{4}+\xi R v_{0}^{2}\right\} \\
& +\xi^{2} v_{0}^{2} \int d^{4} x \sqrt{-g(x)} \int d^{4} y \sqrt{-g(y)} R(x)\left(\frac{1}{\square+4 \lambda v_{0}^{2}}\right)_{x, y} R(y)+\ldots,
\end{aligned}
$$

where the dots stand for the third, fourth and higher order terms in the scalar curvature $R$ with the corresponding insertions of the Green functions. Besides the usual local terms, the last (in fact, more precise) version of the induced tree-level gravitational action includes an infinite set of the non-local terms due to the specific non-constant VEV of the scalar field (23) (25). The appearance of the non-local terms in the induced action (30) is remarkable, also, for other reasons. Although the coefficients of these terms are very small compared to the vacuum Einstein-Hilbert term, the non-localities do not mix with the local terms and, in principle, can lead to some physical effects. If considering the low energy SSB phenomena in the framework of the SM, the non-local terms are irrelevant at low energies due to the large value of the mass term $4 \lambda v_{0}^{2}$. But, if we assume that there is an extremely light scalar (e.g. quintessence), whose mass is of the order of the Hubble parameter and which has a potential admitting a SSB, then the non-localities may become relevant and in particular lead to observable consequences. This part of the story has not been explored so far. Hence, in the next sections we will not discuss it and instead concentrate on the quantum one-loop corrections to the induced action (30).

\section{Effective Action and Renormalization}

Here we start to deal with the main subject of our review. At the quantum level the classical action of vacuum (12) is replaced by the Effective Action (EA) $\Gamma\left[g_{\mu \nu}\right]$, which may be defined via path integral

$$
e^{i \Gamma\left[g_{\mu \nu}\right]}=\int \mathcal{D} \phi e^{i S\left[\phi ; g_{\mu \nu}\right]} .
$$

Here $\phi$ is the set of all matter fields and gauge ghosts, $\mathcal{D} \phi$ is the covariant measure of functional integration. The classical action $S\left[\phi ; g_{\mu \nu}\right]$ includes matter fields, interactions between these fields, it depends on the metric (which plays the role of external parameter) and also the classical action of vacuum (12). In the case when the background matter fields are present, the expression (31) should be generalized in a standard way [31. Here we shall mainly deal with the effective action of vacuum and therefore consider the purely metric background. 
The effective action of gravity $\Gamma\left[g_{\mu \nu}\right]$ admits a loop expansion [31]

$$
\Gamma\left[g_{\mu \nu}\right]=S_{v a c}\left[g_{\mu \nu}\right]+\bar{\Gamma}^{(1)}+\bar{\Gamma}^{(2)}+\bar{\Gamma}^{(3)}+\ldots,
$$

The simplest and usually most important 1-loop contribution is given by the expression

$$
\begin{gathered}
\bar{\Gamma}^{(1)}=\frac{i}{2} \operatorname{Tr} \ln \hat{H} \\
\text { where } \quad \hat{H}=\hat{H}(x, y)=\left.\frac{1}{2} \frac{\delta^{2} S\left[\phi, g_{\mu \nu}\right]}{\delta \phi(x) \delta \phi(y)}\right|_{\phi=0}
\end{gathered}
$$

is the bilinear in quantum fields part of the classical action.

The covariance of effective action can be, presumably, established using the same methods which are used for other gauge interactions [125, 60. Furthermore, as we shall discuss later on, there are explicitly covariant calculational methods. The principal known approaches to calculate quantum corrections are listed below.

\subsection{Possible and impossible form of quantum corrections}

It is remarkable that already at this level one can make some essential statements about possible and impossible forms of quantum corrections. The effective action $\Gamma\left[g_{\mu \nu}\right]$ is a well-defined diffeomorphism invariant quantity. As a consequence, $\Gamma\left[g_{\mu \nu}\right]$ can not include odd powers of the metric derivatives. Let us emphasize that this property is not related to the perturbative expansion and is valid independent on whether the effective actionis a local functional of the metric (indeed, it is nonlocal, as we shall see soon). This important property of effective actionholds for any particular metrics, including the cosmological one.

In case one detects, someday, the odd-power behavior in the gravitational solutions, this would be an indication to a certain "new physics", e.g. quintessence, extra dimensions, branes etc. However, it can not be a vacuum quantum effect of known fields on purely metric background. An interesting application to cosmology is that the quantum corrections to the cosmological constant in the late universe, without scalar fields, may start from $H^{2}$ (here $H$ is the Hubble parameter in the late Universe), but not from $H$, because this would mean an odd metric derivative. In particular, this rules out the hypothetical QCD contributions to the vacuum energy suggested in [105].

One can go further and make even stronger affirmative. Which kind of fundamental physics may be relevant for the possible scale dependence of the vacuum energy? Let us notice the relation

$$
\rho_{\Lambda}(\text { observable }) \propto H_{0}^{2} M_{\text {Planck }}^{2}
$$

where $H_{0}$ is the present-day Hubble parameter. The origin of this relation is the recent astronomical data concerning the energy balance of the universe which is dominated by the $(\sim 75 \%)$ Dark Energy (see, e.g., [103]). The energy of vacuum has mass dimension four and if the $\mathcal{O}\left(H_{0}^{2}\right)$

term is absent, the effect of quantum corrections can be only $\mathcal{O}\left(H_{0}^{4}\right) \sim 10^{-168} \mathrm{GeV}^{4}$, that is negligible compared to the critical density of the universe $\rho_{c} \sim 10^{-48} \mathrm{GeV}^{4}$. Hence we can safely 
assume that the quantum correction to the cosmological constant can be either negligible or have the form

$$
\delta \rho_{\Lambda}(\text { observable }) \sim H_{0}^{2} M^{2},
$$

where $M$ is the typical mass in the theory from where the corresponding quantum correction comes. Looking at the numerical part, it becomes clear that any physics below the GUT scale should be irrelevant for the quantum contribution to the vacuum energy. Indeed, for $M=M_{X} \approx$ $10^{16} \mathrm{GeV}$ the ratio between the hypothetical loop contribution and the value of observable $\rho_{\Lambda}$ or $\rho_{c}$ is about six orders of magnitude. This is, in principle, a detectable effect [71], because it produces an essential difference in the density perturbations spectrum. However, already for the Standard Model we have $M=M_{F} \approx 10^{2}-10^{3} \mathrm{GeV}$ and the difference grows up to the astonishing fifty orders of magnitude. For the vacuum effects of QCD the effect is even much weaker. The conclusion is that, contrary to our intuition, the physics below the GUT scale is likely irrelevant for the potential scale dependence of the vacuum energy. Indeed, this consideration may be applied only to the case when quantum corrections to the vacuum energy do depend on the metric derivatives (or, better say, on Hubble parameter). In principle, one can consider the case when the quantum corrections do not have such dependence [109, 18]. We shall give more details about the possible running of vacuum energy density in section 7 .

\subsection{Calculational methods in curved spaces}

In this section we shall discuss the existing practical methods of quantum calculations in curved spaces and their relevance for establishing the general features of renormalization and structure of finite parts of quantum corrections. We shall avoid the detailed historical considerations which can be found in [64, 25, 31] and only discuss those aspects which are relevant for the consequent sections. We shall start by discussing the three methods (flat-space Feynman diagrams, local momentum representation and Schwinger-DeWitt method) which enable one to establish the general structure of renormalization in curved space-time.

- Feynman diagrams for the perturbations on flat background $h_{\mu \nu}=g_{\mu \nu}-\eta_{\mu \nu}$. Very important early works in this area have been done using this method [121, 134]. In particular, these calculations have shown, for the first time, the necessity of higher derivative terms (13) for renormalizability and the general structure of finite quantum corrections, for both massive and massless cases.

The contributions to the vacuum effective action correspond to the diagrams with internal loops of matter fields and with external lines of the $h_{\mu \nu}$ field. One can expand the action $S[\Phi, g]$ such that the propagators and vertices of all the fields (quantum and background) are the usual ones in the flat space-time. The internal lines of all the diagrams are only those of the matter fields, while external lines are both of matter and gravitational field $h_{\mu \nu}$. As a result, any flatspace digram gives rise to the infinite set of diagrams, with increasing number of the background fields tails. An example of such set is depicted in Figure 1.

Due to the non-polynomial nature of gravity, every flat-space diagram is producing an infinite number of diagrams with external $h_{\mu \nu}$ tails, including an infinite number of divergent diagrams. In order to establish the structure of possible divergences, one has to impose covariance which does not follow automatically from the calculations. Then the number of relevant diagrams 


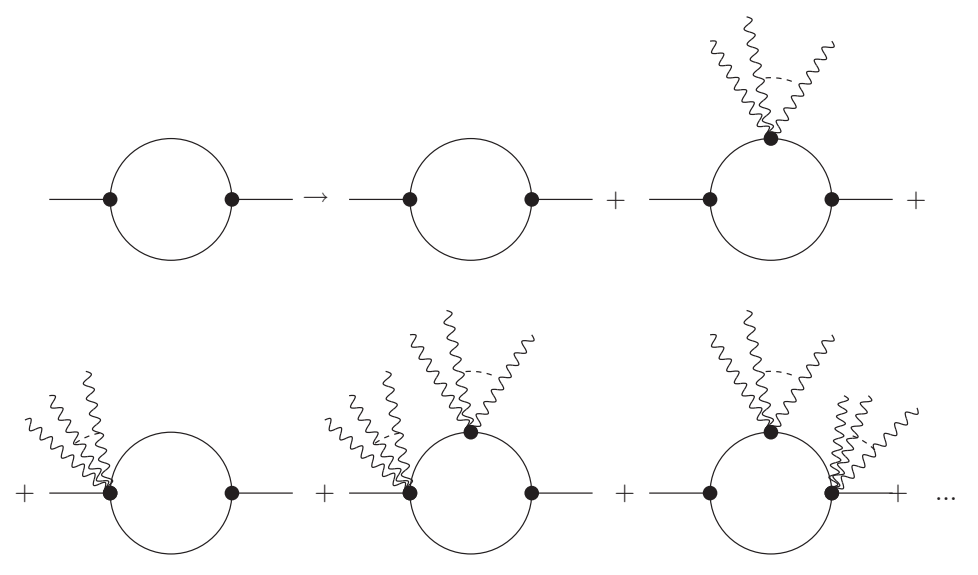

Figure 1: The straight lines correspond to the matter (in this case scalar with $\lambda \varphi^{3}$ interaction) field, and wavy lines to the external metric. A single diagram in flat space-time generates an infinite set of families of diagrams in curved space-time. The first of these generated diagrams is exactly the one in the flat space-time, and the rest have external gravity lines.

becomes finite, making the analysis of renormalization rather simple. The shortcomings of this method are the lack of explicit covariance, difficulties in practical calculations and interpreting the results. However, there is a serious benefit coming from the fact that, in the framework of this method, we deal with the usual flat-space QFT. According to the general theorems of the renormalization theory (see, e.g., [125, 60] and [131]) the divergences in QFT can be removed by the local covariant counterterms. So, let us notice that the necessary counterterms in curved space are local ones.

- The simplest of the known covariant methods is based on the local momentum representation [34]. In general, the use of momentum representation is not possible in curved spaces, however one can use normal coordinates (see, e.g. [98 for the introduction) and perform loop calculations in the tangent space corresponding to a given point $P\left(x_{(0)}^{\mu}\right)$. One can express all quantities of interest, such as free and interaction parts of the classical Lagrangian in form of the power series in $y^{\mu}=x^{\mu}-x_{(0)}^{\mu}$. Due to the special choice of coordinates, the coefficients of this series are components of curvature tensor and its covariant derivatives in the point $P$. The local momentum representation can be used for both propagator and vertices and finally enables one to derive local quantities such as counterterms. For instance the first order expansion for the propagator of a massive non-minimal scalar field has the form

$$
G(k)=\frac{1}{k^{2}+m^{2}}-\frac{1}{3} \frac{(1-3 \xi) R}{\left(k^{2}+m^{2}\right)^{2}}+\frac{2}{3} \frac{R^{\mu \nu} k_{\mu} k_{\nu}}{\left(k^{2}+m^{2}\right)^{3}}+\mathcal{O}\left(k^{-3}\right) .
$$

Similar expansions can be constructed for spinor and vector fields and also for the interaction vertices. Taking the locality of the counterterms into account it is obvious that, after replacing such expansions into Feynman diagrams, the structure of divergences will be as follows. The zero order terms will produce the divergences which are direct covariant generalizations of the ones in flat space-time. Furthermore, the quadratically divergent in flat space diagrams will produce logarithmic divergences (and therefore counterterms) linear in curvature tensor. Due to the 
dimensional reasons and gauge invariance, there are only two types of such terms. The first one is the Einstein-Hilbert type counterterm, with the coefficient proportional to the square of the mass of the quantum field. The second one is the non-minimal scalar-curvature interaction term which really shows up in the interacting scalar theory. Finally, in the next order in curvature there will be the fourth derivative counterterms. They can be constructed from the metric only, because matter fields are dimensional. The most important observation is that, if the original QFT in flat space is a renormalizable theory, starting from the third order in curvatures there will be only finite contributions.

Let us notice that the locality of the counterterms follows from the previous $h_{\mu \nu}=g_{\mu \nu}-\eta_{\mu \nu}$ approach and from the universality of the vacuum EA. The information provided by the two methods which we briefly described above enables one to establish the general structure of renormalization in curved space-time. Another advantage is that these methods can be applied at higher loops. However, for the practical one-loop calculations we better apply another approach.

- Schwinger-DeWitt expansion [129] is the most useful method for calculating divergences and related quantities. The key point is the representation of the $\operatorname{Tr} \ln \hat{H}=\ln \operatorname{Det} \hat{H}$ via the proper time integral

$$
\begin{gathered}
\frac{i}{2} \operatorname{Tr} \ln \hat{H}=-\frac{i}{2} \operatorname{Tr} \int_{0}^{\infty} \frac{d s}{s} e^{-i s \hat{H}} \\
\text { where } \quad e^{-i s \hat{H}}=\hat{U}_{(}\left(x, x^{\prime} ; s\right)=\hat{U}_{0}\left(x, x^{\prime} ; s\right) \sum_{k=0}^{\infty}(i s)^{k} \hat{a}_{k}\left(x, x^{\prime}\right) .
\end{gathered}
$$

$\hat{a}_{k}\left(x, x^{\prime}\right)$ are Schwinger-DeWitt coefficients, while the expression for $\hat{U}_{0}\left(x, x^{\prime} ; s\right)$ has the form

$$
\hat{U}_{0}\left(x, x^{\prime} ; s\right)=\frac{1}{(4 \pi i s)^{n / 2}} \mathcal{D}^{1 / 2}\left(x, x^{\prime}\right) \exp \left\{-\frac{\sigma\left(x, x^{\prime}\right)}{2 i s}-i m^{2} s\right\} .
$$

Here $\sigma\left(x, x^{\prime}\right)$ is the geodesic distance between $x$ and $x^{\prime}$ and

$$
\mathcal{D}\left(x, x^{\prime}\right)=\operatorname{det}\left[-\partial_{\mu} \partial_{\nu} \sigma\left(x, x^{\prime}\right)\right]
$$

is the Van Vleck-Morett determinant. Further details of the standard Schwinger-DeWitt technique can be found, e.g., in [129]. A very powerful generalization, which enables one to derive 1-loop divergences for a variety of quantum theories on curved background and also for various models of quantum gravity, has been developed in [15] (see also references therein). A comprehensive review on the effective action method and in particular generalizations of the Schwinger-DeWitt technique has been recently given in [124].

\subsection{One-loop divergences and renormalization}

In the framework of the Schwinger-DeWitt technique the UV limit corresponds to the lower $s=0$ limit in the proper-time integral (38). The regularizations of this integral can be performed in different ways. The most common is the special version of dimensional regularization [27, 15], but one can use also Zeldovich-Starobinsky approach [134, an equivalent adiabatic regularization [89], point-splitting [40] or even covariant cut-off [7] regularizations. In the four 
dimensional case the $\hat{a}_{0}$-coefficient of (38) corresponds to the quartic diveregence and the $\hat{a}_{1^{-}}$ coefficient corresponds to the quadratic divergence. It is well-known that the cancelation of these divergences may be related to the fine-tunings in imposing the renormalization conditions. This may be lead to difficulties in such cases as hierarchy problem in the Standard Model or the Cosmological Constant problem (which is, actually, also a hierarchy problem [110]) but we will not deal with these issues here. The reason is that, despite the fine tuning is unnatural, in the case of quadratic or quartic divergences it has no apparent concern for the form of quantum corrections which we are interested in.

The most important for us are logarithmic divergences which are proportional to the "magic" coefficient $\hat{a}_{2}=\operatorname{Tr} \lim _{x^{\prime} \rightarrow x} \hat{a}_{2}\left(x, x^{\prime}\right)$ of (38). The logarithmic divergences define such important notions as renormalization group $\beta$-functions and anomalies. The form of $\hat{a}_{2}$ in the vacuum sector is

$$
\hat{a}_{2}=\int d^{4} x \sqrt{g}\left\{\beta_{\Lambda}+\beta_{E} R+\beta_{1} C^{2}+\beta_{2} E+\beta_{3} \square R+\beta_{4} R^{2}\right\} .
$$

wheret4

$$
\begin{aligned}
(4 \pi)^{2} \beta_{\Lambda} & =\frac{1}{2} N_{2} m_{s}^{4}-2 N_{f} m_{f}^{4}, \\
(4 \pi)^{2} \beta_{E} & =N_{s} m_{s}^{2}\left(\xi-\frac{1}{6}\right)+\frac{N_{f} m_{f}^{2}}{3}, \\
(4 \pi)^{2} \beta_{4} & =\frac{N_{s}}{2}\left(\xi-\frac{1}{6}\right)^{2}, \\
(4 \pi)^{2} \beta_{1} & =\frac{1}{120} N_{s}+\frac{1}{20} N_{f}+\frac{1}{10} N_{v}=\omega, \\
(4 \pi)^{2} \beta_{2} & =-\frac{1}{360} N_{s}-\frac{11}{360} N_{f}-\frac{31}{180} N_{v}=b, \\
(4 \pi)^{2} \beta_{3} & =\frac{1}{180} N_{s}+\frac{1}{30} N_{f}-\frac{1}{10} N_{v}=c .
\end{aligned}
$$

Using these formulas, one can write the total expression for the divergent part of the one-loop effective action of vacuum for the theory involving $N_{s}$ real scalars, $N_{f}$ Dirac spinors and $N_{v}$ massless vectors

$$
\bar{\Gamma}_{d i v}^{(1)}=-\frac{1}{n-4} \int d^{4} x \sqrt{-g}\left\{\beta_{1} C^{2}+\beta_{2} E+\beta_{4} R^{2}+\beta_{3} \square R+\beta_{E} R+\beta_{\Lambda}\right\},
$$

In (43) we used the notations of dimensional regularization, but of course the leading logarithmic divergence is regularization independent.

Let us make a few observations concerning the expression for the one-loop divergences (43).

i) The divergences have exactly the form which follows from the general arguments presented above. All high derivative terms of dimension four, Einstein-Hilbert term and cosmological constant are necessary to have a renormalizable theory of matter on curved background. Let us remark that in case the interacting scalar is present, the non-minimal term $\xi R \varphi^{2}$ is also necessary [31] (see the references to the original papers therein).

\footnotetext{
${ }^{4}$ We are introducing the double notations $\beta_{1,2,3}=(\omega, b, c) /(4 \pi)^{2}$. This will prove useful in the sections devoted to conformal anomaly and its applications.
} 
ii) According to the consideration presented above, at any loop order the divergences of the gauge theory in curved space-time are of the same form as the non-minimal classical action with the vacuum term or, in other words, of the same form as one-loop expressions. The only difference is the coefficients of the divergent terms, which start depending on the couplings at higher loops (see, e.g., 68]). Therefore the theory formulated above is multiplicatively renormalizable in curved space-time. At any loop order the counterterms can be removed by renormalizing the full set of parameters of the theory, including couplings, masses, $\xi$ and vacuum parameters. There is no need to renormalize the external metric field.

iii) The one-loop divergences are given by an algebraic sum of the contributions from a free scalar, fermion and vector fields. The reason is that, as we have already mentioned above, each of the Feynman diagrams which we take into account consists of the single loop of a matter field with external tails of a gravity field. No matter vertices show up at this level.

iv) In the massless case only the higher derivative counterterms emerge. This means the global conformal symmetry holds in the one-loop divergences. As we shall see below, however, this symmetry is violated in the finite part of effective action.

v) The non-minimal parameter $\xi$ affects the one-loop divergences, but it can be only seen in the combination $\xi-1 / 6$. As we already know, the value $\xi=1 / 6$ corresponds, in the massless case, to the special version of the scalar theory which possesses the local conformal symmetry. Furthermore, the unique non-conformal counterterm, in the massless case, $\int \sqrt{-g} R^{2}$, has coefficient $(\xi-1 / 6)^{2}$. This means the one-loop divergences are conformal invariant if the original theory is invariant. More precise is to say that the four-dimensional coefficient of the pole term satisfies the conformal Noether identity (18).

vi) All features described in the previous points can be explained in a systematic way. But there are some amazing things in the expressions (42) which are still waiting to be explained. It is easy to see that the contributions of scalars, fermions and vectors to the $\beta$-functions $\beta_{1}$ and $\beta_{2}$ show a universality of signs. The ones to $\beta_{1}$ are all positive and the ones to $\beta_{2}$ are all negative. As we shall see later on, the universality of the signs of $\beta_{2}$ has great importance for the Starobinsky inflationary model. The mentioned sign rules have nothing to do with the Grassmann parity of the fields and remain an unexplained occurrence. It is even more mysterious that the higher derivative conformal fields (scalars and fermions) constructed so far [100, 54, 21] always produce opposite signs (compared to the usual scalar, fermion and vector) of the contributions to both $\beta$-functions.

vii) Coming back to the heat kernel expansion (39) one can notice that the finite terms start from $\operatorname{Tr} \lim _{x^{\prime} \rightarrow x} \hat{a}_{k}\left(x, x^{\prime}\right), \quad k \geq 3$ and have the form of local covariant expressions with growing powers of metric derivatives. For instance, $a_{3}$ has the $R_{\ldots}^{3}$-type and $R_{\ldots} \square R_{\ldots}$-type terms. The general expressions are known for $\hat{a}_{3}$ and $\hat{a}_{4}$ [59, 10].

\section{$3.4 \overline{\mathrm{MS}}$ scheme - based renormalization group}

One can use the multiplicative renormalizability of the QFT in curved space-time to formulate the renormalization group equations for all quantum fields and parameters. As usual, the simplest version of the renormalization group can be constructed in the framework of the minimal subtraction $(\overline{\mathrm{MS}})$ renormalization scheme [86, 32, 119]. The detailed exposition of the $\overline{\mathrm{MS}}$ scheme - based renormalization group method in curved space-time can be found in [31. 
Here we shall only give brief account of this method.

Consider the quantum theory of the fields $\Phi$ on the background of classical metric $g_{\mu \nu}$. The full set of parameters of the theory will be denoted by $P$. For example, in case of SM or GUT like QFT, $\Phi$ includes fermions, Yang-Mills fields and scalars and $P$ includes gauge, Yukawa and scalar self-interaction couplings, non-minimal parameters $\xi$ and the parameters of the vacuum action (12), including Newton and cosmological constants. The dimensions of $\Phi$ and $P$ will be denoted as $d_{\Phi}$ and $d_{P}$, correspondingly.

For the sake of definiteness we assume the dimensional regularization. The $\overline{\mathrm{MS}}$-scheme renormalization group equation for the effective action means that the last is independent on the dimensional renormalization parameter $\mu$

$$
\mu \frac{d \Gamma}{d \mu}=\left\{\mu \frac{\partial}{\partial \mu}+\beta_{P}(n) \frac{\partial}{\partial P}+\int d^{n} x \gamma_{\Phi}(n) \frac{\delta}{\delta \Phi(x)}\right\} \Gamma\left[g_{\alpha \beta}, \Phi, P, n, \mu\right]=0,
$$

where we used the standard notations for the renormalization group $\beta$ and $\gamma$ - functions in $n$-dimensional space-time

$$
\beta_{P}(n)=\mu \frac{d P}{d \mu}, \quad \gamma_{\Phi}(n)=\mu \frac{d \Phi}{d \mu},
$$

while the usual $\beta$-functions correspond to the limit $n \rightarrow 4$. Let us notice that these formulas are essentially the same as in the flat space-time. The main difference is the presence of $g_{\mu \nu}(x)$, playing the role of external parameter. As a result there are several qualitatively new effective charges, such as $\xi$ and vacuum parameters.

The eq. (44) is a formally universal renormalization group equation which can be used for different purposes, depending on the physical interpretation of $\mu$. For example, in order to consider the short distance limit, we can perform a global rescaling of all quantities, including metric and obtain another identity, which is independent on (44)

$$
\Gamma\left[g_{\alpha \beta}, \Phi, P, n, \mu\right]=\Gamma\left[e^{-2 \tau} g_{\alpha \beta}, e^{d_{\Phi} \tau} \Phi, e^{d_{P} \tau} P, n, e^{\tau} \mu\right] .
$$

After being considered together, (44) and (46) produce the general solution of the form

$$
\Gamma\left[g_{\alpha \beta} e^{-2 \tau}, \Phi, P, n, \mu\right]=\Gamma\left[g_{\alpha \beta}, \Phi(\tau), P(\tau), n, \mu\right],
$$

where the effective charges $P(\tau)$ and $\Phi(\tau)$ satisfy the renormalization group equations

$$
\frac{d \Phi}{d \tau}=\left(\gamma_{\Phi}-d_{\Phi}\right) \Phi, \quad \frac{d P}{d \tau}=\beta_{P}-P d_{P}
$$

The limit $\tau \rightarrow \infty$ corresponds to the short distances and, due to

$$
R_{\mu \nu \alpha \beta}^{2} \rightarrow R_{\mu \nu \alpha \beta}^{2} e^{4 \tau}, \quad R_{\alpha \beta}^{2} \rightarrow R_{\alpha \beta}^{2} e^{4 \tau}, \quad R \rightarrow R e^{2 \tau}, \ldots
$$

to the limit of greater curvatures. In this respect it is equivalent to the standard rescaling of momenta in the flat-space quantum field theory. On the other hand, the application of (47) and (48) to particular situations needs a special attention. For example, let us consider the exponential inflation. The time-dependence of the metric

$$
g_{\alpha \beta} \rightarrow g_{\alpha \beta} \cdot e^{H t}, \quad H=\text { const }
$$


looks similar to the rescaling (47). However, inflation does not fit the $\overline{\mathrm{MS}}$-based renormalization group, because the scalar curvature $R=-12 H^{2}$ does not behave according to (49). The origin of the difference is that the parameter of the global rescaling $\tau$ is a constant, while the time $t$ in (50) is a coordinate and therefore the transformation (50) is a local one.

In order to understand the physical significance of the renormalization group running in curved space one has to attribute some physical sense to the parameter $\mu$. At this point one has several option. Let us formulate the above question in a different way: which terms in the effective action can be parametrized by the $\overline{\mathrm{MS}}$-scheme renormalization group in curved space? Remember that the standard interpretation of the $\overline{\mathrm{MS}}$-scheme renormalization group in flat space is related to the high energy limit in the momentum subtraction scheme of renormalization. In this case, in order to get the terms in effective action which are behind the renormalization group, one has to replace $\ln \left(\mu^{2} / \mu_{0}^{2}\right)$ by the expression $\ln \left(p^{2} / p_{0}^{2}\right)$, where $p^{2}$ is the square of the momentum. In the coordinate representation this means we have to introduce the formfactor $\ln \left(\square / m^{2}\right)$. For example, in case of QED, the corresponding term looks like

$$
\beta F^{\mu \nu} \ln \left(\frac{\square}{m^{2}}\right) F_{\mu \nu} .
$$

This procedure can be indeed generalized to the case of a curved space. The expected term is, for instance,

$$
\beta_{1} C^{\mu \nu \alpha \beta} \ln \left(\frac{\square}{m^{2}}\right) C_{\mu \nu \alpha \beta}
$$

for the case of the Weyl term in the action of vacuum (12). Of course, the d'Alembertian operator in (52) is the covariant one. Later on, in section 6, we shall confirm the presence of the term (52) by a direct calculation [61]. The $\overline{\mathrm{MS}}$-scheme based procedure described above can be successfully applied to derive the quantum corrections to the classical action of gravity and, e.g., scalar field [33, 31] (see also [45] for an alternative consideration). At the same time, this approach meets obvious difficulties when applied to the running of the Newton or cosmological constants [61, 14, 85]. In these cases the formfactor can not be simply inserted into the action, because the d'Alembertian operator acting on the cosmological constant gives zero. Similarly, in the Einstein-Hilbert term, the formfactor can only produce the total derivatives, or superficial terms, which do not affect the equations of motion for gravity. We shall come back to these arguments in section 7 .

Can we state that the identification of $\mu^{2}$ with $\square$ is a universal tool making the $\overline{\mathrm{MS}}$-scheme results physically relevant? Unfortunately, the problem is not really solved by the analogy with QED, because in gravity, according to the relations (49) one can identify $\mu$ with other metric-dependent quantities.

For example, the scalar curvature has the same global scaling law as the d'Alembertian operator $\square$. In this way we can construct the curvature-controlled version of renormalization group. It is interesting that the corresponding expressions in the effective action can be also obtained by special resummation of the Schwinger-deWitt series [90, 91]. From the general perspective the identification of $\mu^{2}$ with the scalar curvature is as legitimate as its identification with $\square$ and moreover it has the following two advantages: the possibility to write down the quantum correction to the cosmological and Newton constants and the closeness to the most natural identification of $\mu$ with the Hubble parameter in the cosmological setting [11, 111]. One 
has to remember, however, that anyone of these identifications is no more than a particular model for an unknown complete effective action. An obvious manifestation of this feature is a vast ambiguity which one meets in the resummed and truncated expressions used in [91. It is important to remember that the renormalization group is a method to parametrize the scaling dependence of the effective action. In case of gravity it is especially difficult to introduce a physically consistent and universal renormalization group, in particular because definition of the energy of the gravitational field is a nontrivial problem (see, e.g., 44] and references therein).

The solution of the renormalization group equations (48) has been explored for different QFT models in curved space [31] (see further references therein). The equations for couplings and masses of the quantum matter fields are exactly the same as in flat space-time [39]. The qualitatively new elements are the equations for the nonminimal parameters $\xi$ and for the parameters of the vacuum action $\Lambda, G, a_{1,2,3,4}$. The one-loop $\beta_{\xi}$ is always proportional to the difference $\xi-1 / 6$, such that the conformal value $\xi=1 / 6$ is a universal fixed point of the renormalization group trajectory in the theory with scalar fields. The coefficient of proportionality depends on the model. For some gauge models it leads to the UV asymptotic conformal invariance [28] and for other models the conformal value is the IR fixed point. The general conditions for the asymptotic behavior of $\xi$ have been found in [29]. Indeed at higher loops the $\beta_{\xi}$ is not proportional to $\xi-1 / 6$ factor anymore [68]. The related ambiguities lead to the quantum inconsistency of the conformal invariant theory [7] beyond the one loop approximation.

Finally, there is one subtlety in the physical application of the renormalization group equations for the cosmological and Newton constants (42)

$$
(4 \pi)^{2} \mu \frac{d}{d \mu}\left(\frac{\Lambda}{8 \pi G}\right)=\beta_{\Lambda}-\frac{\Lambda}{2 \pi G}, \quad \mu \frac{d}{d \mu}\left(\frac{1}{16 \pi G}\right)=\beta_{E}-\frac{1}{8 \pi G} .
$$

Do we need the second terms in the r.h.s. of these two equations? In order to address this question one has to remember that $\Lambda$ and $G$ gain physical sense when they are inserted into the Einstein equations

$$
R_{\mu \nu}-\frac{1}{2} R g_{\mu \nu}=8 \pi G T_{\mu \nu}+\Lambda g_{\mu \nu}
$$

The second terms of (53) reflect the classical dimension of $\Lambda$ and $G$, but other components of (54) also have their own dimensions. As a result the classical scaling terms do cancel and, for the applications, one has to use only anomalous dimensions, that are the $\beta$-functions terms.

\section{Conformal anomaly and anomaly-induced EA}

In general, there is no way to calculate the vacuum effective action completely. In order to understand why this is so, let us remember that already in the Schwinger-DeWitt expansion we observe an infinite series in curvature tensor and its derivatives. On the top of that, the renormalization group and analogy with QED indicates, as we have discussed in the previous section, the presence of an infinite amount of non-local insertions. So, the task of deriving the full effective action does not look realistic. It is remarkable that there is a class of the four-dimensional theories for which we are able to derive exactly the non-local terms in $\bar{\Gamma}^{(1)}$. The word "exactly" here means we can do it at the one-loop level and only on the very special 
backgrounds. Despite of these restrictions, the situation deserves this wording, as a way to emphasize an enormous difference with the situation typical for other kinds of theories.

In this section we consider the anomalous violation of local conformal symmetry in the case of quantum matter on classical curved background. The conformal anomaly enables one to derive the anomaly-induced effective action. In the consequent section we shall discuss the applications of anomaly and of the induced effective action.

\subsection{Derivation of conformal anomaly}

Quantum anomaly is a typical phenomenon in a situation where the original theory has more than one symmetry. The origin of anomalies is the renormalization procedure [97]. The anomaly shows up if there is no regularization which preserves all symmetries at the quantum level. After the divergences are subtracted, in the finite part of the effective action some of the symmetries are getting broken. In our case the theory has general covariance and local conformal symmetry, and the last is broken by quantum corrections.

Consider quantization of free massless conformal invariant matter fields denoted by $\Phi$, on classical gravitational background. As before, we assume that the set of quantized matter fields $\Phi$ includes $N_{s}$ scalars (all with $\xi=1 / 6$ ), $N_{f}$ fermions and $N_{v}$ vectors. We denote $k_{\Phi}$ the conformal weight of the field.

At the one-loop level it is sufficient to consider the simplified vacuum action

$$
S_{\text {conf. vac }}=\int d^{4} x \sqrt{-g}\left\{a_{1} C^{2}+a_{2} E+a_{3} \square R\right\} .
$$

Let us emphasize that it is not wrong to supplement the last expression by the Einstein-Hilbert action, cosmological constant or the $\int \sqrt{-g} R^{2}$-term. The action (55) can be seen as a part of classical action which is a subject of an infinite renormalization at the one-loop level. Beyond the one-loop approximation the $\int \sqrt{-g} R^{2}$ term is also necessary, because the conformal theory becomes inconsistent [7].

The Noether identity for the local conformal symmetry has the form (18) and can be interpreted as $T_{\mu}^{\mu}=0$ on shell (see section 2). At quantum level $S_{v a c}\left(g_{\mu \nu}\right)$ has to be replaced by the effective action of vacuum $\Gamma_{v a c}\left(g_{\mu \nu}\right)$. As we already know, its divergent part is

$$
\Gamma_{d i v}=\frac{1}{\varepsilon} \int d^{4} x \sqrt{g}\left\{\beta_{1} C^{2}+\beta_{2} E+\beta_{3} \square R\right\}
$$

where $\varepsilon=(n-4)^{-1}$ in dimensional regularization. In the case of global conformal symmetry, the renormalization group method or $\zeta$-regularization tell us [69, 32, 31]

$$
<T_{\mu}^{\mu}>=\beta_{1} C^{2}+\beta_{2} E+a^{\prime} \square R
$$

where $a^{\prime}=\beta_{3}$. In the case of local conformal invariance there is an ambiguity in the parameter $a^{\prime}[25,48,6]$. This issue has been explained recently in [7] and reviewed in [106], so we will not discuss it in full details here. Qualitatively the result is that the ambiguity is always equivalent to the freedom to add the $\int \sqrt{-g} R^{2}$-term to the classical action.

One can derive the conformal anomaly in different ways, mainly due to the choice of regularization schemes [49, 40, 25, 7]. We shall follow [49] and [7, 106], using dimensional regularization. 
We are interested in the vacuum effects and therefore, at the one-loop level, can restrict consideration by the free fields case. The expression for divergences is (56) with the $\beta$-functions defined in (42). The renormalized one-loop effective action has the form

$$
\Gamma_{R}=S+\bar{\Gamma}+\Delta S
$$

where $\bar{\Gamma}=\bar{\Gamma}_{d i v}+\bar{\Gamma}_{f i n}$ is the naive quantum correction to the classical action and $\Delta S$ is an infinite local counterterm which is called to cancel the divergent part of $\bar{\Gamma}$ (56). Indeed $\Delta S$ is the only source of the noninvariance of the effective action, since naive (but divergent) contributions of quantum matter fields are conformal. The anomalous trace is therefore equal to

$$
T=<T_{\mu}^{\mu}>=-\left.\frac{2}{\sqrt{-g}} g_{\mu \nu} \frac{\delta \Gamma_{R}}{\delta g_{\mu \nu}}\right|_{n=4}=-\left.\frac{2}{\sqrt{-g}} g_{\mu \nu} \frac{\delta \Delta S}{\delta g_{\mu \nu}}\right|_{n=4} .
$$

The calculation of this expression can be done, in a most simple way, as follows. Let us change the parametrization of the metric to

$$
g_{\mu \nu}=\bar{g}_{\mu \nu} \cdot e^{2 \sigma}, \quad \sigma=\sigma(x),
$$

where $\bar{g}_{\mu \nu}$ is the fiducial metric with fixed determinant. There is a useful relation

$$
-\frac{2}{\sqrt{-g}} g_{\mu \nu} \frac{\delta A\left[g_{\mu \nu}\right]}{\delta g_{\mu \nu}}=-\left.\frac{1}{\sqrt{-\bar{g}}} e^{-4 \sigma} \frac{\delta A\left[\bar{g}_{\mu \nu} e^{2 \sigma}\right]}{\delta \sigma}\right|_{\bar{g}_{\mu \nu \rightarrow g_{\mu \nu}, \sigma \rightarrow 0}} .
$$

At that point we need transformation laws for the structures presented in (56). They can be found, for instance, in [38], so we will not reproduce these formulas here. For instance, for the square of the Weyl tensor we have

$$
\int d^{n} x \sqrt{-g} C^{2}(n)=\int d^{n} x \sqrt{-\bar{g}} e^{(n-4) \sigma} \bar{C}^{2}(n) .
$$

All other expressions of our interest (56) have the same factor $e^{(n-4) \sigma}$ and, on the top of that, some extra terms with derivatives of $\sigma(x)$. For all terms which are not total derivatives, these terms are irrelevant due to the procedure (61).

In the simplest case of global conformal factor $\sigma=\lambda=$ const we immediately arrive at the expression (57) with $a^{\prime}=\beta_{3}$. However in the local case $\sigma=\sigma(x)$ the situation is more complicated. It is worth mentioning that the left hand side in (61) gives zero when applied to the integral of the total derivative term $\int \sqrt{-g} \square R$. On the other hand, the value of $a^{\prime}$ can be modified by adding a finite term

$$
S_{+}=\alpha \int d^{4} x \sqrt{-g} R^{2}
$$

to the classical action (12), due to the identity

$$
-\frac{2}{\sqrt{-g}} g_{\mu \nu} \frac{\delta}{\delta g_{\mu \nu}} \int d^{4} x \sqrt{-g} R^{2}=12 \square R .
$$

The last formula can be derived either directly or through the eq. (61). The same effect can be achieved by the term $\int \sqrt{-g} R_{\mu \nu}^{2}$,

$$
-\frac{2}{\sqrt{-g}} g_{\mu \nu} \frac{\delta}{\delta g_{\mu \nu}} \int d^{4} x \sqrt{-g} R_{\mu \nu} R^{\mu \nu}=4 \square R
$$


and also by the term $\int \sqrt{-g} R_{\mu \nu \alpha \beta}^{2}$. For the sake of simplicity, below we shall discuss only the term $\int \sqrt{-g} R^{2}$.

One may think that adding the classical non-conformal term (63) has nothing to do with the quantum corrections. However, consider in more details how to apply the procedure (59) to the counterterm of the $\int \sqrt{-g} C^{2}$-type. The point is that the Weyl tensor depends on the dimension $d$, in particular its square is

$$
C^{2}(d)=C_{\alpha \beta \mu \nu}(d) C^{\alpha \beta \mu \nu}(d)=R_{\mu \nu \alpha \beta}^{2}-\frac{4}{d-2} R_{\mu \nu}^{2}+\frac{2}{(d-1)(d-2)} R^{2} .
$$

When defining the corresponding counterterm

$$
\Delta S_{C}=\frac{\beta_{1}}{n-4} \int \sqrt{-g} C^{2}(d)
$$

one can choose the Weyl tensor with $d=n+\gamma(n-4)$, where $\gamma$ is an arbitrary number. For any such $d$ the counterterm is local, it cancels the divergent part of effective action and the renormalization is multiplicative in the $R_{\mu \nu \alpha \beta}^{2}, R_{\mu \nu}^{2}$ and $R^{2}$ basis. However, the anomalous $\square R$ term depends on the choice of $\gamma$. Hence at this point we meet an arbitrariness. The particular choice $\gamma=-1$ has been done in [49]. In this case we arrive at

$$
T\left(C^{2}\right)=\left.\frac{2}{\sqrt{-g}} g_{\mu \nu} \frac{\delta}{\delta g_{\mu \nu}} \frac{\mu^{n-4}}{n-4} \int d^{n} x \sqrt{-g} \beta_{1} C^{2}(4)\right|_{n \rightarrow 4}=C^{2}-\frac{2}{3} \square R .
$$

For scalars and spinors the result is identical for the global and local conformal symmetries violation. In case of vectors there is no such equivalence and, moreover, this is only one particular case of the possible counterterms. Finally, it is easy to see that the difference between the counterterms (67) with different $d$ is equivalent to the finite $\int \sqrt{-g} R^{2}$-term. Qualitatively similar ambiguity takes place in the covariant Pauli-Villars regularization [7]. Finally, the anomaly is given by (57), but there is an ambiguity in the coefficient $a^{\prime}$. If most of regularization schemes $a^{\prime}=\beta_{3}$ but, in general, fixing this coefficient requires a special renormalization condition [6, 106].

\subsection{Anomaly-induced action}

One can use conformal anomaly to construct the equation for the finite part of the 1-loop correction to the effective action (the notations are according to (42))

$$
\frac{2}{\sqrt{-g}} g_{\mu \nu} \frac{\delta \bar{\Gamma}_{i n d}}{\delta g_{\mu \nu}}=\omega C^{2}+b E+c \square R
$$

The solution of this equation is straightforward [100] (see also generalizations for the theory with torsion [35] and with a scalar field [108]). Here we shall follow a bit more detailed exposition given in [106]. The simplest possibility is to parametrize metric as in (62), separating the conformal factor $\sigma(x)$ and rewrite the eq. (69) using (61). The solution for the effective action is

$$
\begin{aligned}
\bar{\Gamma} & =S_{c}\left[\bar{g}_{\mu \nu}\right]+\int d^{4} x \sqrt{-\bar{g}}\left\{\omega \sigma \bar{C}^{2}+b \sigma\left(\bar{E}-\frac{2}{3} \square \bar{R}\right)+2 b \sigma \bar{\Delta}_{4} \sigma\right. \\
& \left.\left.-\frac{1}{12}\left(c+\frac{2}{3} b\right)\left[\bar{R}-6(\bar{\nabla} \sigma)^{2}-(\square \sigma)\right]^{2}\right)\right\}
\end{aligned}
$$


where $S_{c}\left[\bar{g}_{\mu \nu}\right]=S_{c}\left[g_{\mu \nu}\right]$ is an unknown functional of the metric, which serves as an integration constant for the eq. (69). The merit of the solution (70) is its simplicity, but it is not covariant or, in other words, it is not expressed in terms of original metric $g_{\mu \nu}$. It proves useful to obtain the covariant solution [100, 107]. Let us establish the following relations [100] (see also [38] for details):

$$
\begin{gathered}
\sqrt{-g} C^{2}=\sqrt{-\bar{g}} \bar{C}^{2}, \quad \sqrt{-\bar{g}} \bar{\Delta}_{4}=\sqrt{-g} \Delta_{4}, \\
\sqrt{-g}\left(E-\frac{2}{3} \square R\right)=\sqrt{-\bar{g}}\left(\bar{E}-\frac{2}{3} \square \bar{R}+4 \bar{\Delta}_{4} \sigma\right),
\end{gathered}
$$

where $\Delta_{4}$ is a fourth derivative conformally covariant operator acting on dimensionless scalar

$$
\Delta_{4}=\square^{2}+2 R^{\mu \nu} \nabla_{\mu} \nabla_{\nu}-\frac{2}{3} R \square+\frac{1}{3} R_{; \mu} \nabla^{\mu}
$$

and also introduce the Green function for this operator $\Delta_{4, x} G(x, y)=\delta(x, y)$. Using these formulas and (61) we find, for any $A=A\left(\bar{g}_{\mu \nu}, \sigma\right)$, the relation

$$
\left.\frac{\delta}{\delta \sigma(y)} \int d^{4} x \sqrt{-g(x)} A\left(E-\frac{2}{3} \square R\right)\right|_{g_{\mu \nu}=\bar{g}_{\mu \nu}}=4 \sqrt{-\bar{g}} \bar{\Delta}_{4} A=4 \sqrt{-g} \Delta_{4} A .
$$

Using this relation it is easy to find the term in the effective action, which is responsible for $T_{\omega}=-\omega C^{2}$,

$$
\Gamma_{\omega}=\frac{\omega}{4} \int d^{4} x \sqrt{-g(x)} \int d^{4} y \sqrt{-g(y)} C^{2}(x) G(x, y)\left(E-\frac{2}{3} \square R\right)_{y} .
$$

Similarly one can find the term which produces $T_{b}=b\left(E-\frac{2}{3} \square R\right)$

$$
\Gamma_{b}=\frac{b}{8} \int d^{4} x \sqrt{-g(x)} \int d^{4} y \sqrt{-g(y)}\left(E-\frac{2}{3} \square R\right)_{x} G(x, y)\left(E-\frac{2}{3} \square R\right)_{y} .
$$

The third constituent of the induced action follows from eq. (64)

$$
\Gamma_{c}=-\frac{3 c+2 b}{36} \int d^{4} x \sqrt{-g(x)} R^{2}(x) .
$$

The covariant solution of eq. (69) is a sum of the expressions (75), (176) and (77).

The anomaly-induced action presented in a local form using auxiliary scalar fields. After using the classical equations of motion for these fields and replacing them back to the action we come to the original non-local expressions. In order to construct the local covariant representation, the action should be presented in a symmetric form

$$
\begin{aligned}
\Gamma_{\omega, b} & =\int d^{4} x \sqrt{-g(x)} \int d^{4} y \sqrt{-g(y)}\left(E-\frac{2}{3} \square R\right)_{x} G(x, y)\left[\frac{\omega}{4} C^{2}-\frac{b}{8}\left(E-\frac{2}{3} \square R\right)\right]_{y} \\
& =-\frac{b}{8} \int d^{4} x d^{4} y \sqrt{g(y) g(x)}\left[\left(E-\frac{2}{3} \square R\right)-\frac{\omega}{b} C^{2}\right]_{x} G(x, y)\left[\left(E-\frac{2}{3} \square R\right)-\frac{\omega}{b} C^{2}\right]_{y} \\
& -\frac{1}{2} \int d^{4} x d^{4} y \sqrt{g(y) g(x)}\left(\frac{\omega}{2 \sqrt{-b}} C^{2}\right)_{x} G(x, y)\left(\frac{\omega}{2 \sqrt{-b}} C^{2}\right)_{y}
\end{aligned}
$$


The last two terms are appropriate objects for rewriting them using the auxiliary fields. In this way we arrive at the following final expression for the anomaly generated effective action of gravity.

$$
\begin{aligned}
\bar{\Gamma} & =S_{c}\left[g_{\mu \nu}\right]-\frac{3 c+2 b}{36} \int d^{4} x \sqrt{-g(x)} R^{2}(x) \\
& +\int d^{4} x \sqrt{-g(x)}\left\{\frac{1}{2} \varphi \Delta_{4} \varphi-\frac{1}{2} \psi \Delta_{4} \psi+\varphi\left[k_{1} C^{2}+k_{2}\left(E-\frac{2}{3} \square R\right)\right]+l_{1} \psi C^{2}\right\},
\end{aligned}
$$

where

$$
k_{1}=-l_{1}=-\frac{\omega}{2 \sqrt{-b}} \quad \text { and } \quad k_{2}=\frac{\sqrt{-b}}{2}
$$

Some important remarks are in order.

1) The local covariant form (79) is dynamically equivalent to the non-local one (78), (77). The complete definition of the Cauchy problem in the theory with the non-local action requires defining the boundary conditions for the Green functions $G(x, y)$, independently in the two terms (75) and (76). The same can be achieved, in the local version, by imposing the boundary conditions for the two auxiliary fields $\varphi$ and $\psi$.

2) The local form of effective action with two auxiliary scalars (79) has been introduced in the paper [107. Qualitatively similar manner of introducing second scalar has been suggested later on in [79]. The kinetic term for the auxiliary field $\varphi$ is positive while for $\sigma$ it was negative. For $\psi$ the kinetic term has negative sign. The wrong sign does not lead to problems here, because both fields are auxiliary and do not propagate independently.

3) We introduced the new structure $\int C_{x}^{2} G(x, y) C_{y}^{2}$ into the action, despite it was not directly produced by anomaly. This term is indeed conformal invariant and therefore its emergence may be viewed as a simple redefinition of the conformal invariant functional $S_{c}\left[g_{\mu \nu}\right]$. On the other hand, writing the non-conformal terms in the symmetric form (78), we have modified the four point function in a very essential way. Therefore, introducing the mentioned conformal term we have just restored the basic structure of the terms generated by anomaly. For this reason, the second auxiliary scalar [107] represents a natural element of writing the induced action in a local form.

\subsection{Light massive fields}

Before we start to discuss the applications of conformal anomaly and of the anomalyinduced action, let us show how the same approach may be useful for exploring the effective action of light massive fields. In section 6 we shall also consider the case of heavy massive fields. In order to understand why these two cases must be treated separately, let us come back and look at the Schwinger-DeWitt expansion (39). It is easy to see that it corresponds to the growing powers in metric derivatives. Due to the covariance, this is actually an expansion in the positive powers in curvatures and their covariant derivatives. The dimension is compensating by the negative powers of the mass $m$ of the quantum field. Indeed, such expansion is going to be efficient is the curvature invariants are much smaller than $m^{2}$. In other words this is an approximation for the "heavy" fields case. However, there are physical situations where we need another approximations, for instance for the case of light fields, where curvature invariants 
satisfy $\left|R_{\ldots . .}\right| \ll m^{2}$, or for an intermediate regime, where these two quantities are of the same order of magnitude. It is remarkable that we have no regular approximations for these two cases and, in the last case, there is no available method at all. However, for the light fields, there is one method, suggested in [108] and slightly generalized in [95].

Let us consider the theory where the conformal invariance of scalar and fermion actions is violated only by the masses of these fields and by the Einstein-Hilbert action. The idea is to treat the masses as perturbations with respect to the massless theory. Originally, there is no local conformal symmetry and hence one can not use the conformal anomaly to derive quantum corrections. But, this can be changed if we apply the conformal parametrization of the massive theory. The transformation is similar to the one used long ago in the cosmon model [93] and in GR [42]. The calculations perform in the following three steps:

1) Conformization of the theory;

2) Derivation of anomaly and anomaly-induced effective action of the new theory;

3) Coming back to the original parametrization.

Let us replace the dimensional parameters in both matter and gravitational sectors by the powers of a new auxiliary scalar field $\chi$ according to

$$
\begin{array}{ccc}
m_{s}^{2} \rightarrow \frac{m_{s}^{2}}{M^{2}} \chi^{2} \quad(\text { scalar mass }), \quad m_{f} \rightarrow \frac{m_{f}}{M} \chi & \text { (fermion mass) }, \\
\frac{1}{16 \pi G} R \rightarrow \frac{M_{P}^{2}}{16 \pi M^{2}}\left[R \chi^{2}+6(\partial \chi)^{2}\right], & \Lambda \rightarrow \frac{\Lambda}{M^{2}} \chi^{2} .
\end{array}
$$

Here $M$ is a new dimensional parameter. In order to provide the local conformal invariance we postulate that the auxiliary field $\chi$ transforms as $\chi \rightarrow \chi e^{-\sigma}, \sigma=\sigma(x)$. Under the procedure (81), the massive terms for the matter fields are replaced by Yukawa and scalar interactions involving the new auxiliary scalar $\chi$. The new action is conformal invariant in both matter and gravitational sectors. The classical Noether identity for the new vacuum action has the form

$$
\mathcal{T}=\left(-\frac{2}{\sqrt{-g}} g_{\mu \nu} \frac{\delta}{\delta g_{\mu \nu}}+\frac{1}{\sqrt{-g}} \chi \frac{\delta}{\delta \chi}\right) S_{v a c}\left[g_{\mu \nu}, \chi\right]=0 .
$$

The conformal invariance is violated by a conformal anomaly and one can derive the effective action of the background fields $g_{\mu \nu}, \chi$ using the anomaly-induced effective action scheme. The divergences of the theory in the conformal representation have the form

$$
\Gamma_{\text {div }}^{(1)}=-\frac{\mu^{n-4}}{(n-4)} \int d^{n} x \sqrt{-g}\left\{w C^{2}+b E+c \nabla^{2} R+\frac{f}{M^{2}}\left[R \chi^{2}+6(\partial \chi)^{2}\right]+\frac{g}{M^{4}} \chi^{4}\right\},
$$

where the coefficients $w, b$ and $c$ are given by Eq. (42) and

$$
f=\frac{1}{3(4 \pi)^{2}} \sum_{f} N_{f} m_{f}^{2}, \quad g=\frac{1}{2(4 \pi)^{2}} \sum_{s} N_{s} m_{s}^{4}-\frac{2}{(4 \pi)^{2}} \sum_{f} N_{f} m_{f}^{4} .
$$

Here the sums are taken over massive fermion $f$ and scalar $s$ fields with the masses $m_{f}$ and $m_{s}$ correspondingly, $N_{f}$ and $N_{s}$ being multiplicities.

The conformal anomaly is proportional to the integrand of (83)

$$
<\mathcal{T}>=-\left\{w C^{2}+b E+c \nabla^{2} R+\frac{f}{M^{2}}\left[R \chi^{2}+6(\partial \chi)^{2}\right]+\frac{g}{M^{4}} \chi^{4}\right\} .
$$


In the standard way, described in section 5.1, one can derive the anomaly-induced effective action of the background fields $g_{\mu \nu}$ and $\chi$

$$
\begin{aligned}
\Gamma_{i n d} & =S_{c}\left[g_{\mu \nu}, \chi\right]+\int d^{4} x \sqrt{-\bar{g}}\left\{w \bar{C}^{2} \sigma+b\left(\bar{E}-\frac{2}{3} \bar{\nabla}^{2} \bar{R}\right) \sigma+2 b \sigma \bar{\Delta} \sigma+\right. \\
& \left.+\frac{f}{M^{2}}\left[\bar{R} \bar{\chi}^{2}+6(\partial \bar{\chi})^{2}\right] \sigma+\frac{g}{M^{4}} \bar{\chi}^{4} \sigma\right\}-\frac{3 c+2 b}{36} \int d^{4} x \sqrt{-g} R^{2} .
\end{aligned}
$$

The last step is to fix the conformal unitary gauge $\chi=\bar{\chi} e^{-\sigma}=M$, such that the classical Einstein-Hilbert and cosmological terms acquire their standard form

$$
\begin{aligned}
\Gamma & =S_{H D}+\bar{\Gamma}_{i n d}\left[\bar{g}_{\mu \nu}, \sigma\right] \\
& -\int d^{4} x \sqrt{-\bar{g}} e^{2 \sigma}\left[\bar{R}+6(\bar{\nabla} \sigma)^{2}\right] \cdot\left(\frac{1}{16 \pi G}-f \cdot \sigma\right)-\int d^{4} x \sqrt{-\bar{g}} e^{4 \sigma} \cdot\left(\frac{\Lambda}{8 \pi G}-g \cdot \sigma\right),
\end{aligned}
$$

where $\bar{\Gamma}_{i n d}\left[\bar{g}_{\mu \nu}, \sigma\right]$ has been defined in (70).

Let us discuss the level of reliability behind the calculation of the effective action (87). First of all, it is easy to see that this is not an exact result, even for the FLRW-like metric. The reason is that the integration constant $S_{c}\left[g_{\mu \nu}, \chi\right]$ in (86) depends on the intermediate scalar $\chi$ and, after fixing $\chi \rightarrow M, S_{c}\left[g_{\mu \nu}, M\right]$ is not conformal invariant functional anymore. The second observation is that the higher-derivative part of the Eq. (87) is identical to that for the massless fields. Let us remember that the one-loop effective action is given by $(i / 2) \ln \operatorname{Det} \hat{H}$ of some operator, depending on the background metric [130]. The Det $\hat{H}$ can be seen as a product of the eigenvalues of the operator $\hat{H}$, depending on the corresponding eigenfunctions (see, e.g., [50]). When we are adding the masses of the fields, there are two distinct effects: modified eigenvalues due to the masses and also modified eigenfunctions. It is obvious that the calculation presented above takes into account only the first effect, that is why the higher derivative sector does not change. Perhaps the second effect manifests itself in the $S_{c}\left[g_{\mu \nu}, M\right]$-term, but this part is out of our control.

In order to understand better the approximation which is assumed in our calculus, let us notice a strong link between the anomaly-induced effective action Eq.(87) and the quantum corrections coming from the renormalization group. The expansion of the homogeneous, isotropic universe means a conformal transformation of the metric $g_{\mu \nu}(t)=\bar{g}_{\mu \nu} \exp [\sigma(\eta)]$. On the other hand, the renormalization group in curved space-time corresponds to the scale transformation of the metric $g_{\mu \nu} \rightarrow g_{\mu \nu} \cdot e^{-2 \tau}$ simultaneous with the inverse transformation of all dimensional quantities [31]. For any $\mu$ we have $\mu \rightarrow \mu \cdot e^{\tau}$. One can compare the $\sigma$-dependence of the anomaly-induced effective action (87) and the $\tau$-dependence of the renormalization-group improved classical action

$$
S_{\text {vacuum }}[P(\tau)]
$$

where $P=\left(a_{1,2,3,4}, G, \Lambda\right)$ denote vacuum parameters of the theory and $P(\tau)=P_{0}+\beta_{P} \tau$. The expression (88) is a leading-log approximation for the solution of the renormalization group equation for the effective action [31]

$$
\Gamma\left[e^{-2 \tau} g_{\alpha \beta}, \Phi_{i}, P, \mu\right]=\Gamma\left[g_{\alpha \beta}, \Phi_{i}(\tau), P(\tau), \mu\right],
$$


where $\Phi_{i}$ are matter fields. It is easy to see that (87) becomes completely equivalent to (88) if we set $\sigma=$ const. The coefficient $f$ is a factor of the $\beta$-function for the inverse Newton constant $1 / 16 \pi G$ and the coefficient $g$ is a factor of the $\beta$-function for the cosmological term $\Lambda / 8 \pi G$. Indeed, (89) can be considered as a generalization of the renormalization group improved classical action (88). After all, one can consider Eq. (87) as a leading-log approximation for the effective action for the massive fields. This approximation picks up the logarithmic quantum corrections and is reliable in the high energy region where masses of the fields are much smaller than the Hubble constant $H$. The anomaly-induced expression may be even seen as a $\overline{\mathrm{MS}}$-scheme based local version of renormalization group [113], but the consistent formulation of such local renormalization group is not available yet.

\section{Applications of the anomaly-induced action}

The most important areas of application for the quantum corrections to the action of vacuum are cosmology and the black-hole physics. In both cases the anomaly-induced effective action described in the previous section is behind the most well-established results, such as Hawking radiation and Starobinsky model of inflation. In this part of our review we shall give a very brief account of these two subjects. We shall follow the original publications [12, 52, 95] where one can find more detailed considerations.

\subsection{Vacuum states in the vicinity of a black hole}

At the classical level, the black hole does not emit radiation, but such emission can take place if we take quantum effects into account. After the theoretical discovery of the black hole evaporation by Hawking [70, the same result has been obtained from analytical estimates of $\left\langle T_{\mu \nu}\right\rangle$ for matter fields propagating in a fixed static Schwarzschild black hole geometry. Indeed, the black hole evaporation phenomenon has not been confirmed experimentally. In the situation when the complete information on the vacuum state or, equivalently, on the effective action of vacuum is unavailable, one can not be absolutely certain that such evaporation really takes place. However, all known methods converge predicting such quantum radiation. The completely consistent approach would involve the simultaneous solution of the following two problems: solving equations of motion derived from the effective action and derivation of the quantum evaporation of the black hole. However, the practical calculations which have been performed so far, are based on the reduced approach, when the quantum effects are estimated on the background of the purely classical solution. Here we shall use the same approximation [12].

The idea to derive the Hawking effect starting from the effective action of vacuum is natural. The main problem here is to choose the appropriate method for evaluating the effective action. For example, in [84] (see also [13]) the starting expression has been derived starting from the two-dimensional conformal anomaly in the dimensionally reduced (from $d=4$ ) theory. As a result there are serious difficulties and the fit to the standard result requested some artificial procedure related with introducing an effective potential of the conformal factor of the metric. Similar situation takes place in recent works [123, where the consideration was based on a new complicated approach to deriving the effective action of vacuum.

Let us notice that no one of existing techniques for the effective action of vacuum is perfect 
in a sense there is always an ambiguity. The unique case when we can arrive at the exact one-loop result is the conformally trivial (e.g., FRW) metric, but this is definitely different from the black-hole situation. Then, since it is known the Hawking effect is closely related to the four-dimensional conformal anomaly [41, it is natural to expect the best fit with the traditional methods if starting from the anomaly-induced effective action. Indeed, this approach really enables one to classify the vacuum states in a somehow clear way and reproduce the standard result for the Hawking radiation [12]. Moreover, the analogy with the black hole vacuum turns out to be very fruitful for the investigation of the gravitational waves in a anomaly-induced background [52], where we achieve a close fit with the well known result of [114] and with the consequent calculations [72].

Consider the application of the anomaly-induced effective action (179) to the analysis of the quantum effects in the vicinity of a black hole. We shall see that the different vacuum states of the black hole (Boulware, Hartle-Hawking and Unruh) correspond to the different choices of initial conditions for the two auxiliary fields [12]. The result can be generalized for the ReissnerNordstrom spacetime case [4]. Let us stress that such classification can not be accomplished by using only one field $\varphi$. Therefore the correspondence with other approaches to Hawking radiation confirms that our considerations about introducing the second auxiliary scalar $\psi$ are correct.

Detailed analytical and numerical investigations, based on the analysis of $\left\langle T_{\mu \nu}\right\rangle$ in the classical black hole background have been performed in [37]. One of the fundamental properties of this background is the existence of three different vacuum quantum states, which are defined as follows:

i) The Boulware vacuum $|B\rangle[26$ ] is obtained by choosing in and out modes to be positive frequency with respect to the Killing vector $\partial_{t}$ of the Schwarzschild metric (2). This state reproduce the Minkowski vacuum $|M\rangle$ asymptotically, in the limit $r \rightarrow \infty$ we find $\left\langle B\left|T_{\mu \nu}\right| B\right\rangle \sim$ $1 / r^{6}$. At the same time the behavior of the same average value on the horizon $r=2 M$ is divergent in a free falling frame. In this situation, for the classical observables, one can choose another coordinates, e.g. (44), such that the horizon becomes free of the singularities. In quantum theory this corresponds to choosing another vacuum state.

ii) For the Unruh vacuum $|U\rangle$ [120] the in modes have positive frequency with respect to $\partial_{t}$, while the out modes are taken to be positive frequency with respect to Kruskal's $U=$ $-4 M e^{-u / 4 M}$ (44). The value $\left\langle U\left|T_{\mu \nu}\right| U\right\rangle$ is regular on the future event horizon but not on the past one. Asymptotically in the future $\left\langle U\left|T_{\mu \nu}\right| U\right\rangle$ has the form of a flux of radiation at the Hawking temperature $T_{H}=1 / 8 \pi M$. Hence this vacuum state is the most appropriate to discuss evaporation of black holes formed by gravitational collapse of matter.

iii) The Israel-Hartle-Hawking $|H\rangle$ vacuum state [66] is obtained by choosing in modes to be positive frequency with respect to the Kruskal's coordinate $V=4 M e^{v / 4 M}$ and out modes positive frequency with respect to the affine parameter along the past horizon. $\left\langle H\left|T_{\mu \nu}\right| H\right\rangle$ for $r \rightarrow \infty$ describes a thermal bath of radiation at the Hawking temperature $T_{H}$. This vacuum state corresponds to a black hole in equilibrium with a surrounding thermal bath. Asymptotically both in the future and the past $|H\rangle \neq|M\rangle$.

The existence of three distinct vacuum states in quantum theory looks natural, in sense they reflect distinct choices of coordinates systems and construction of different in and out modes with 
respect to the corresponding coordinates. The main difference between classical and quantum theories is that, in the first case we know how to transform the coordinates. So, the natural question is how to perform the transition between different vacuum states $|H\rangle,|B\rangle$ and $|U\rangle$ ?

The anomaly-induced part of the effective action do not make any reference to a particular quantum state. Therefore one has to look for the non-universal part of effective action in order to implement one or another state. We remember that the solution (178) is exact only for the conformally-trivial metric, such as the Friedmann-Robertson-Walker (FRW) one. In all other cases the conformal invariant functional $S_{c}\left[g_{\mu \nu}\right]$ is a source of uncertainty. Furthermore, in order to apply the quantum correction (79) one has to fix the extended set of boundary conditions, including the ones for the auxiliary scalar fields $\varphi$ and $\psi$. It turns out that the identification of the Boulware and Unruh vacuum states can be performed, in the leading approximation, by choosing an appropriate initial and boundary conditions for the auxiliary scalars. In order to achieve the Israel-Hartle-Hawking one has to make an additional adjustment of the conformal functional. We will not go into details here, because one can easily find them in [12] (see also [13] where the more simple effective two-dimensional case has been treated) and present just the list of main results.

The equations of motion for the auxiliary fields $\varphi$ and $\psi$ have the form

$$
\begin{aligned}
-\frac{1}{\sqrt{-g}} \frac{\delta \bar{\Gamma}}{\delta \phi} & =\Delta_{4} \phi+k_{1} C^{2}+k_{2}\left(E-\frac{2}{3} \square R\right)=0, \\
\frac{1}{\sqrt{-g}} \frac{\delta \bar{\Gamma}}{\delta \psi} & =-\Delta_{4} \psi+l_{1} C^{2}=0,
\end{aligned}
$$

where we used the notations (80). In the Ricci-flat space the operator $\Delta_{4}$ boils down to $\square^{2}$, such that we get

$$
\square^{2} \phi=\frac{\alpha(G M)^{2}}{r^{6}}
$$

where $\alpha=-48\left(k_{1}+k_{2}\right)$. The equation for $\psi$ can be obtained by trading $\alpha \rightarrow \beta=48 l_{1}$. Until the end of this subsection we shall set the Newton constant $G=1$. It proves useful to introduce the traceless tensor $K_{\mu \nu}$ (an explicit form of this object can be found in [12])

$$
K_{\mu \nu}(\varphi)=-\frac{1}{\sqrt{-g}} \frac{\delta}{\delta g^{\mu \nu}} \int d^{4} x \sqrt{-g} \varphi \Delta_{4} \varphi
$$

then

$$
\begin{aligned}
-\frac{2}{\sqrt{-g}} \frac{\delta \Gamma}{\delta g^{\mu \nu}} & =\left\langle S_{\mu \nu}\right\rangle=K_{\mu \nu}(\varphi)-K_{\mu \nu}(\psi)-8 \nabla^{\lambda} \nabla^{\tau} Z R_{\mu \lambda \nu \tau}+g_{\mu \nu} Z R_{\rho \sigma \alpha \beta}^{2} \\
& -4 Z R_{\mu \rho \lambda \tau} R_{\nu}^{\rho \lambda \tau}-\frac{4 k_{2}}{3}\left[\left(\nabla_{\mu} \nabla_{\nu} \square \varphi\right)-g_{\mu \nu}\left(\square^{2} \varphi\right)\right]+\ldots,
\end{aligned}
$$

where we omitted those term which vanish on the Ricci flat background such as the Schwarzschild metric. Let us notice that $\left\langle S_{\mu \nu}\right\rangle$ represents only that part of the average Energy-Momentum Tensor which can be restored from anomaly, being in general different from the unknown complete quantity $\left\langle T_{\mu \nu}\right\rangle$.

The strategy for identifying the given vacuum state is as follows. One solves the equations (90) for the auxiliary fields and replace the solutions into (93). These solutions will always 
depend on the set of integration constants and one can try to find the "correct" ones to identify $\left\langle V\left|T_{\mu \nu}\right| V\right\rangle$ for the given vacuum state $|V\rangle$, being it $|V\rangle=|B\rangle,|U\rangle$ or $|H\rangle$.

The general solution for $\varphi$ has the form $\phi(r, t)=d \cdot t+w(r)$, where $w(r)$ satisfies the equation

$$
\begin{aligned}
\frac{d w}{d r} & =\frac{B}{3} r+\frac{2 M B}{3}-\frac{A}{6}-\frac{\alpha}{72 M}+\frac{1}{r-2 M}\left(\frac{4}{3} B M^{2}+\frac{C}{2 M}-A M-\frac{\alpha}{24}\right) \\
& -\frac{C}{2 M} \frac{1}{r}+\ln r\left[-\frac{\alpha M}{18} \frac{1}{r(r-2 M)}-\frac{r^{2}}{3(r-2 M)}\left(\frac{A}{2 M}-\frac{\alpha}{48 M^{2}}\right)\right] \\
& +\frac{\left(r^{3}-8 M^{3}\right) \ln (r-2 M)}{3 r(r-2 M)}\left(\frac{A}{2 M}-\frac{\alpha}{48 M^{2}}\right)
\end{aligned}
$$

and $(d, A, B, C)$ are constants that specify the homogeneous solution $\square^{2} \phi=0$ and hence the quantum state. For $\psi$ we have a similar solution, but with another set of integration constants $\left(d^{\prime}, A^{\prime}, B^{\prime}, C^{\prime}\right)$. It is important that these two sets are independent on each other, due to the independence of $\varphi$ and $\psi$.

In the case of a Boulware state $|B\rangle$ we request $|B\rangle \rightarrow|M\rangle$ when $r \rightarrow \infty$. For the auxiliary scalars in the Minkowski vacuum we can safely set $\varphi=\psi=0$. This asymptotic conditions and also cancelation of singular terms in the solution of (94) enables one to fix all constants and we arrive at the asymptotic expressions 12 ]

$$
\left\langle B\left|S_{\mu}^{\nu}\right| B\right\rangle \rightarrow \frac{1}{2} \frac{\alpha^{2}-\beta^{2}}{(24)^{2}} \frac{1}{(2 M)^{4}(1-2 M / r)^{2}}\left(\begin{array}{cccc}
-1 & 0 & 0 & 0 \\
0 & 1 / 3 & 0 & 0 \\
0 & 0 & 1 / 3 & 0 \\
0 & 0 & 0 & 1 / 3
\end{array}\right)
$$

for $r \rightarrow 2 M$ and

$$
\left\langle B\left|S_{\mu}^{\nu}\right| B\right\rangle \propto \mathcal{O}\left(r^{-6}\right) \quad \text { for } \quad r \rightarrow \infty .
$$

This behaviors perfectly reproduce the one which is observed in the framework of other methods [37. Namely, we have $\mathcal{O}\left(r^{-6}\right)$ in the space infinity and the quadratic divergence $\mathcal{O}\left((1-2 M / r)^{-2}\right)$ near the horizon in the Boulware case.

Another success of our method comes in the case of the Unruh vacuum. Using the same treatment of auxiliary fields, but choosing another values of the constants $(d, A, B, C)$ and $\left(d^{\prime}, A^{\prime}, B^{\prime}, C^{\prime}\right)$, we meet, near the horizon, the following asymptotic behavior [12]:

$$
\left\langle U\left|S_{a}{ }^{b}\right| U\right\rangle \sim \frac{\alpha^{2}-\beta^{2}}{2\left(48 M^{2}\right)^{2}}\left(\begin{array}{cc}
1 / f & -1 \\
1 / f^{2} & -1 / f
\end{array}\right), \quad r \rightarrow 2 M, \quad \text { where } \quad a, b=r, t,
$$

which is regular on the future horizon. The asymptotic form at the space infinity is

$$
\left\langle U\left|S_{\mu}{ }^{\nu}\right| U\right\rangle \rightarrow \frac{\alpha^{2}-\beta^{2}}{2 r^{2}(24 M)^{2}}\left(\begin{array}{cccc}
-1 & -1 & 0 & 0 \\
1 & 1 & 0 & 0 \\
0 & 0 & 0 & 0 \\
0 & 0 & 0 & 0
\end{array}\right), \quad r \rightarrow \infty .
$$


The results of eqs. (97), (98) are in exact agreement with the standard ones on Hawking radiation [37, 130], once the luminosity $L$ of the radiating black hole is identified with

$$
\frac{L}{4 \pi}=\frac{\left(\alpha^{2}-\beta^{2}\right)}{2(24 M)^{2}}
$$

A little bit more complicated situation takes place for the Hartle-Hawking vacuum, where one should not only properly choose the initial conditions but also fine-tune the coefficient $l_{1}$ to order to achieve correspondence with the results achieved by other methods. From the general perspective this situation looks somehow natural because, as we already explained earlier, the unknown conformal invariant functional $S_{c}$ is relevant for the spherically symmetric metric. The modification of $l_{1}$ is nothing else but the adjustment of $S_{c}$. So, we can consider as a kind of luck that the most essential part of the classification of the vacuum states can be performed by the use of the initial conditions for $\varphi$ and $\psi$, such that only in the case of $|H\rangle$ we are forced to modify the conformal term.

\subsection{Modified Starobinsky model.}

Another interesting application of the anomaly-induced action is the Modified Starobinsky Model or anomaly-induced inflation [52, 108, 95]. Let us follow [108, 95] and first consider quantum effects of massless fields, that is use the effective action (70). At the second stage we shall take masses of the fields into account, that means we shall use the action (87).

As a first step consider an empty space, when matter is absent. There are two equivalent ways to arrive at the cosmological solution of the theory with quantum corrections: using the (0-0)-component [53, 114] or via the anomaly-induced effective action [35, 31]. Let us choose the last option. The resulting equation has, for $k=0$ FRW metric, the following form:

$$
\frac{\dddot{a}}{a}+\frac{3 \ddot{a} \dddot{a}}{a^{2}}+\frac{\ddot{a}^{2}}{a^{2}}-\left(5+\frac{4 b}{c}\right) \frac{\ddot{a} \dot{a}^{2}}{a^{3}}-\frac{M_{P}^{2}}{8 \pi c}\left(\frac{\ddot{a}}{a}+\frac{\dot{a}^{2}}{a^{2}}-\frac{2 \Lambda}{3}\right)=0,
$$

where the coefficients $b, c$ are defined in (42). The last equation does not depend on the coefficient $\omega$, because the Weyl tensor vanish for the FRW background. The equation (100) has a remarkable particular solution

$$
a(t)=a_{0} \cdot \exp (H t)
$$

where

$$
H=\frac{M_{P}}{\sqrt{-32 \pi b}}\left(1 \pm \sqrt{1+\frac{64 \pi b}{3} \frac{\Lambda}{M_{P}^{2}}}\right)^{1 / 2}
$$

As far as $\Lambda \ll M_{P}^{2}$, we meet two very different values of $H$ (we consider $\Lambda>0$ )

$$
H_{c} \approx \sqrt{\frac{\Lambda}{3}} \quad \text { and } \quad H_{S} \approx \frac{M_{P}}{\sqrt{-16 \pi b}} .
$$

\footnotetext{
${ }^{5}$ The cases $k= \pm 1$ are quite similar 95 , and we do not consider them here.
} 
The solution with $H_{c}$ is the one of the theory without quantum corrections. The second value $H_{S}$ corresponds to the inflationary solution of Starobinsky [114. Let us notice that the sign of $b$ is negative independent on the particle content (42). Let us remark that the expression "particle content" here corresponds to the degrees of freedom contributing to the vacuum effective action in the virtual loops and has nothing to do with the matter content of the universe.

Further understanding of the inflationary solution requires analyzing its stability properties. The phase portrait of the theory may look very different depending on the sign of the coefficient $c$, that is on the coefficient of the local $\int \sqrt{-g} R^{2}$-term [114, 95]. The inflationary solution is stable for a positive $c$ and is unstable in the case $c<0$. In the last case there are several stable points (attractors), one of which corresponds to the usual $a \sim t^{1 / 2}$ solution. It is interesting that the existence of the exponential solution is possible due to the non-local and universal $\Gamma_{b}$-term in the anomaly-induced effective action, while the stability depends on the local $\int \sqrt{-g} R^{2}$-term only.

The Starobinsky model looks appealing, in particular because it has a solid QFT background and there is no need to introduce a special inflaton field. The original model [114] is based on the unstable solution $c<0$. In this case one has to choose the initial conditions in a very special way. First of all, the initial point must be very close to the exact exponential solution (101), such that the inflation lasts long enough. Moreover, one has to provide that, after the inflationary phase ends, the Universe will approach the attractor corresponding to the FRW solution, and not to other, physically unacceptable, attractors. All the matter content of the Universe is created after the inflation ends through the decay of the massive degree of freedom induced by anomaly. Many interesting aspects of this model, including gravitational waves and problem of singularities, has been investigated starting from the papers [114, 122, 3]. The first investigation of inflationary density perturbations has been also performed for this model [82].

An alternative way to use the solution (70) [52] is based on a positive value of $c$ and therefore on the stability of the exponential solution at the beginning of inflation. It turns out that the stable inflation is very robust with respect to the choice of initial data [95, providing the following two advantages: First, one does not need to consider an empty universe in the initial state, since the stable inflation "kills" any matter content in a few Planck times [96]. Second, there is no problem with the unphysical solutions of the "run-away" sort, the measure of the corresponding initial data is infinitesimal for $c>0$.

The real problem of the stable $c>0$ inflationary model is to understand the period when the inflation ends. If we stay within the original framework [53, 114, that is consider only massless conformal fields, the stable inflation will be eternal. The modified Starobinsky model solves this problem using the effective quantum field theory approach [108] and in particular the notion of decoupling. Using the relations (42), the condition of stability $c>0$ can be cast into the form

$$
N_{v}<\frac{1}{3} N_{f}+\frac{1}{18} N_{s}
$$

The inflation is stable if there are many scalars and fermions, for a given amount of vectors. We know that in QFT vectors are responsible for the fundamental interactions. It is easy to see that the inequality (104) is not satisfied for the MSM with $N_{v, f, s}=(12,24,4)$. However, it is satisfied for the MSSM with $N_{v, f, s}=(12,32,104)$. The same must be true for any realistic supersymmetric model, because the supersymmetrization of the realistic model implies 
transformation of fermions and scalars into chiral superfields and vectors into vector superfields. Both operations require adding many spinor and scalar superpartners (sparticles) while the vector sector of the theory remains the same.

The transition between stable and unstable inflation can be associated with the soft SUSY breaking. Within this approach the sparticles are supposed to be very heavy compared to the usual particles. The inflation becomes unstable when the typical energy becomes smaller than the mass of the sparticles and they decouple. As before, we associate the typical energy with the Hubble parameter $H$. Let us denote $M_{*}$ the energy scale where the inequality (104) changes its sign to the opposite. The value $H_{S}$ is now seen just as initial value of $H$ and $H=M_{*}$ is the final point of the stable inflation. The transition occurs at the instant $t_{f}$ which is defined as a solution of the equation $H\left(t_{f}\right)=M_{*}$.

The details of decoupling will be considered in the next section. The next problem is to find why the inflation slows down. In fact, this can be discovered at once if we take the masses of the fields into account and use the result (86). The equation of motion for $a(t)$ has the form

$$
\begin{aligned}
\frac{\dddot{a}}{a}+3 \frac{\dot{a}}{a} \frac{\dddot{a}}{a}+\frac{\ddot{a}^{2}}{a^{2}}-\left(5+\frac{4 b}{c}\right) \frac{\ddot{a}}{a} \frac{\dot{a}^{2}}{a^{2}} & -\frac{M_{P}^{2}}{8 \pi c}\left[\left(\frac{\ddot{a}}{a}+\frac{\dot{a}^{2}}{a^{2}}\right) \cdot(1-\tilde{f} \cdot \ln a)-\frac{\tilde{f}}{2} \frac{\dot{a}^{2}}{a^{2}}\right] \\
& +\frac{M_{P}^{2} \Lambda}{12 \pi c}(1-\tilde{g} \cdot \ln a-\tilde{g} / 4)=0 .
\end{aligned}
$$

where we introduced useful notations

$$
\tilde{f}=\frac{16 \pi f}{M_{P}^{2}}=\frac{1}{3 \pi} \sum_{f} \frac{N_{f} m_{f}^{2}}{M_{P}^{2}} ; \quad \tilde{g}=\frac{8 \pi g}{M_{P}^{2} \Lambda}=\frac{1}{4 \pi} \sum_{s} \frac{N_{s} m_{s}^{4}}{M_{P}^{2} \Lambda}-\frac{1}{\pi} \sum_{f} \frac{N_{f} m_{f}^{4}}{M_{P}^{2} \Lambda} .
$$

The leading terms in the eq. (105) are the same as in the massless case (100), but the Planck mass and the cosmological constant to be replaced by the variable expressions

$$
\begin{gathered}
M_{P}^{2} \rightarrow M_{P}^{2}(1-\tilde{f} \ln a), \\
\Lambda M_{P}^{2} \rightarrow \Lambda M_{P}^{2}(1-\tilde{g} \ln a) .
\end{gathered}
$$

Let us assume that, because of supersymmetry, the quantum loops contributions to the cosmological constant do cancel $\tilde{g} \approx 0$ [95. Furthermore, $\Lambda$ itself is negligible at the energy scale of inflation. Then the solution of equation (105) can be obtained by solving (107) in the form

$$
\sigma(t)=\ln a(t)=H_{0} t-\frac{H_{0}^{2}}{4} \tilde{f} t^{2} .
$$

The numerical analysis confirms the parabolic dependence (109) with excellent precision [95]. The total number of the inflationary $e$-folds for different models of the SUSY breaking can be obtained from the relation (109). For example, in the case of MSSM we have $M_{*} \sim 1 T e V$, then $\tilde{f} \sim\left(M_{*} / M_{P}\right)^{2}=10^{-32}$ and the total amount of inflationary $e$-folds is $10^{32}$.

One can use the effective QFT approach to perform additional tests of quantum corrections (70). If we believe to the decoupling idea, the present-day Universe looks like a perfect object to apply the effective approach. In the present-day Universe only the photon should be treated as an active physical degree of freedom and hence, according to (104), $c<0$. Then the explicit analysis of stability performed in [95], shows that the solution $H_{c}$ in eq. (103) is stable if $\Lambda>0$. 

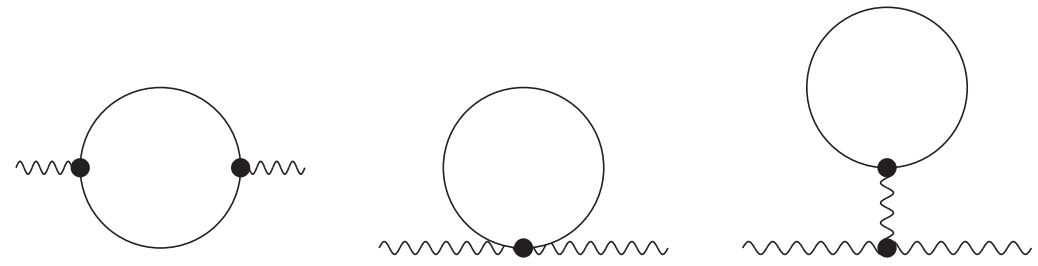

Figure 2: The one-loop diagrams contributing to the polarization operator of gravitons. The matter field loop is connected to external gravitational fields represented by wavy lines.

Furthermore, an additional advantage of the anomaly-induced is the moderate behavior of the gravitational waves or, in other words, restricted production of gravitons [52.

Until now we could see only good points of the anomaly-induced inflation. This model is completely based on the QFT results, it does not require introducing new artificial entities such as inflaton, does not require fine tuning for the action or for initial data, moreover the gravitational waves do not grow too much during the stable phase, making consistent the whole approach based on the homogeneous and isotropic metric. Unfortunately, there is also a real problem with this model. As we already know, the total amount of the inflationary $e$-folds is enormous. However, only the last $60-70$ of these $e$-folds are physically relevant. In order to extract the physical information one needs, therefore, an effective action in the region when $H \propto M_{*}$. However, as we have already discussed above, we have no approximation for this situation, which lies between the light-mass and the heavy-mass extremes. No model exists for the intermediate situation. Until such model will be constructed, the only way to study inflation is through the inflaton approach. However, the very fact that the inflation can be explained by quantum effects of matter fields surely looks interesting and greatly increase the importance of the further study of effective action of massive fields.

\section{Effective action for massive fields}

For a while we have seen that two kinds of information are available about the effective action in curved space. First, we can completely calculate the divergences and remove them using the renormalization procedure. The procedure works perfect in curved space-time, independent on that we do not have access to the complete expression for the effective action or, equivalently, can not obtain the vacuum state of the quantum fields on a classical gravitational background. The key point is that the divergences are given by the well known local expressions and the unknown part of the effective action is non-local. Second, we are able to obtain the non-local part within the anomaly-induced scheme. In this section we shall go further and obtain, in a regular way, some non-local terms for the massive fields theory. We shall present only the main results here, the details can be found in the original papers [61, 20]. 


\subsection{Decoupling theorem for gravity}

As we learned above, the vacuum divergences can be removed by the renormalization of the action (12). This procedure leads to the $\overline{\mathrm{MS}}$ scheme - based renormalization group running, which enables one to restore the high energy asymptotics of the effective action by trading $\ln \mu^{2}$ by $\ln \square[33$, 31]. Now we ask the following questions: are these $\ln \square$ terms really there? And what happens at low energies, when the $\overline{\mathrm{MS}}$ scheme does not work? We know that in QED the main phenomenon at the low-energy regime (we shall call it IR) is the decoupling of massive fields [9]. One can expect something like that in the vacuum gravitational sector, because the general structure of the Feynman diagrams is similar (see, e.g., Fig. 2).

The most obvious way to the decoupling theorem is to derive the effective actions and the $\beta$-functions $\beta_{\lambda}, \beta_{R}, \beta_{1, . ., 4}$ through the $h_{\mu \nu}=g_{\mu \nu}-\eta_{\mu \nu}$ expansion approach [61. This method opens the way for the momentum-subtraction renormalization scheme, that is the standard approach for the Appelquist and Carazzone theorem [9]. A useful alternative to the flat-space expansion is a resummation of the Schwinger-DeWitt series, culminated in the heat kernel solution of [16]. The results obtained within these two methods coincide [61], and for the scalar field we arrive at the following result in the $\mathcal{O}\left(R_{\ldots}^{2}\right)$ approximation:

$$
\begin{aligned}
\bar{\Gamma}_{\text {scalar }}^{(1)} & =\frac{1}{2(4 \pi)^{2}} \int d^{4} x \sqrt{-g}\left\{\frac{m^{4}}{2} \cdot\left(\frac{1}{\epsilon}+\frac{3}{2}\right)+\left(\xi-\frac{1}{6}\right) m^{2} R\left(\frac{1}{\epsilon}+1\right)\right. \\
& \left.+\frac{1}{2} C_{\mu \nu \alpha \beta}\left[\frac{1}{60 \epsilon}+k_{W}(a)\right] C^{\mu \nu \alpha \beta}+R\left[\frac{1}{2 \epsilon}\left(\xi-\frac{1}{6}\right)^{2}+k_{R}(a)\right] R\right\} .
\end{aligned}
$$

Here $\epsilon$ is the parameter of dimensional regularization

$$
\frac{1}{\epsilon}=\frac{2}{4-n}+\ln \left(\frac{4 \pi \mu^{2}}{m^{2}}\right)-\gamma
$$

It is easy to see that the divergences are exactly the same as in the $\overline{\mathrm{MS}}$ scheme (43), (42). The qualitatively new part is encoded into the formfactors (nonlocal insertions produced by quantum corrections), which have the form

$$
\begin{aligned}
k_{W}(a) & =\frac{8 A}{15 a^{4}}+\frac{2}{45 a^{2}}+\frac{1}{150} \\
k_{R}(a) & =A\left(\xi-\frac{1}{6}\right)^{2}-\frac{A}{6}\left(\xi-\frac{1}{6}\right)+\frac{2 A}{3 a^{2}}\left(\xi-\frac{1}{6}\right)+\frac{A}{9 a^{4}}-\frac{A}{18 a^{2}}+\frac{A}{144}+ \\
& +\frac{1}{108 a^{2}}-\frac{7}{2160}+\frac{1}{18}\left(\xi-\frac{1}{6}\right) .
\end{aligned}
$$

Here we used notations

$$
A=1-\frac{1}{a} \ln \left(\frac{2+a}{2-a}\right), \quad a^{2}=\frac{4 \square}{\square-4 m^{2}} .
$$

Similar expressions can be found for massive fermion and vector loops [61 and also for the scalar field background, where the formfactors can be obtained for the $\varphi^{2} R$ and $\varphi^{4}$ terms. In principle, there is a possibility to obtain similar expressions in the third order in curvatures using the corresponding results for the heat kernel [16].

In the flat space the renormalization group scaling is defined as the momentum scaling $p^{2} \rightarrow e^{2 \tau} p^{2}$. In curved space renormalization group may be defined as scaling of metric in the 
coordinate space $g_{\mu \nu} \rightarrow e^{-2 \tau} g_{\mu \nu}$, that is consistent with the flat space limit because $p^{2}=$ $\eta_{\mu \nu} p^{\mu} p^{\nu}$. In the mass-dependent scheme we define the $\beta$-function for the parameter $\lambda$, as

$$
\beta_{\lambda}=-2 p^{2} \frac{\partial \lambda}{\partial p^{2}}
$$

and furthermore identify $p^{2}$ with $-\square$. For the Weyl term we have, following this procedure 61],

$$
\beta_{1}=-\frac{1}{(4 \pi)^{2}}\left(\frac{1}{18 a^{2}}-\frac{1}{180}-\frac{a^{2}-4}{6 a^{4}} A\right) .
$$

From this expression we obtain

$$
\begin{aligned}
\beta_{1}^{U V} & =-\frac{1}{(4 \pi)^{2}} \frac{1}{120}+\mathcal{O}\left(\frac{m^{2}}{p^{2}}\right)=\beta_{1}^{\overline{M S}}+\mathcal{O}\left(\frac{m^{2}}{p^{2}}\right) \\
\text { and } \quad \beta_{1}^{I R} & =-\frac{1}{1680(4 \pi)^{2}} \cdot \frac{p^{2}}{m^{2}}+\mathcal{O}\left(\frac{p^{4}}{m^{4}}\right),
\end{aligned}
$$

that is a quadratic decoupling in agreement with the Appelquist and Carazzone theorem. For $a_{3}$ the situation is similar. The UV regime gives the same result as in (42),

$$
\beta_{3}^{U V}=-\frac{1}{180(4 \pi)^{2}}+\mathcal{O}\left(\frac{m^{2}}{p^{2}}\right)
$$

while the IR limit shows the standard quadratic decoupling

$$
\beta_{3}^{\text {scalar, }, I R}=-\frac{1}{1260(4 \pi)^{2}} \frac{p^{2}}{m^{2}}+\mathcal{O}\left(\frac{p^{4}}{m^{4}}\right) .
$$

An important feature of $\beta_{3}$ is that it changes sign between UV and IR regimes in the models with broken SUSY due to the decoupling of sparticles [61]. As we have seen in the previous section, this property is required for the modified Starobinsky model of inflation.

One can, also, establish the quadratic decoupling in directly in the formfactors. The UV limit corresponds to $a \rightarrow 2$ and the IR limit to $a \rightarrow 0$. It is easy to check that in the IR limit $A \sim-a^{2} / 12 \rightarrow 0$ and therefore in the IR both $k_{W}(a)$ and $k_{R}(a)$ tend to zero as $\mathcal{O}\left(a^{2}\right)$. One can observe the same behavior for the formfactors in the scalar sector $k_{\lambda}(a)$ and $k_{\xi}(a)$ [20]. The gravitational version of the Appelquist and Carazzone theorem holds also in the case of spontaneous symmetry breaking [62], where the analysis is more cumbersome.

\subsection{Massless limit in the massive theory}

As we have seen in the previous section, the dominating phenomenon in the massless theory is the conformal anomaly. It would be quite interesting to obtain anomaly or at least part of it from the second order in curvature expressions such as (110). For this end one has to consider the massless limit. Another interesting aspect of this limit in the specific case of the vector theory with the softly broken gauge invariance is the discontinuity of quantum corrections, which was recently described in details in [30]. Let us consider these two issues here. 


\subsubsection{Anomaly from the heat kernel solution.}

In the massless conformal limit $m^{2}=0, \xi=1 / 6$ we obtain, from the $R\left(k_{R}\right) R$-term, the expression

$$
-\frac{1}{12 \cdot 180(4 \pi)^{2}} \int d^{4} x \sqrt{-g} R^{2},
$$

fitting perfectly with the conformal anomaly [17]. This result can be generalized, also, for massive fermions and vectors. Let us remark that the $R\left(k_{R}\right) R$-term remains non-local even for a very small but non-zero mass and, therefore, it becomes ambiguous only in the precisely massless theory. Let us remark that in the general nonconformal theory an infinite renormalization of the $\int \sqrt{-g} R^{2}$-term is always necessary to make the theory finite. In the conformal massless case this term has to be included in order to fix the finite ambiguity. In this sense there is no $m \rightarrow 0$ discontinuity.

It is also instructive to look at the massless limit of the formfactor for the Weyl term

$$
\begin{aligned}
& \left.\int d^{4} x \sqrt{-g} C_{\mu \nu \alpha \beta}\left(\frac{1}{60 \epsilon}+k_{W}\right) C^{\mu \nu \alpha \beta}\right|_{m \rightarrow 0}= \\
= & \frac{1}{60} \int d^{4} x \sqrt{-g} C_{\mu \nu \alpha \beta}\left\{\left[\frac{2}{4-n}-\ln \left(-\frac{\square}{4 \pi \mu^{2}}\right)\right]+\text { const }\right\} C^{\mu \nu \alpha \beta} .
\end{aligned}
$$

This term defines the leading-log quantum contribution to the propagation of the gravitational wave, that is the transverse traceless part of the gravitational perturbation $h_{\mu \nu}=g_{\mu \nu}-\eta_{\mu \nu}$.

It is easy to see that this expression, also, directly reproduce the Weyl-square term in the conformal anomaly. For this end we have to just use the definition (59) and the relation (61). For the conformally transformed metric $g_{\mu \nu}=\bar{g}_{\mu \nu} \exp (2 \sigma)$, we have

$$
\sqrt{-g} C_{\mu \nu \alpha \beta} C^{\mu \nu \alpha \beta}=\sqrt{-\bar{g}} \bar{C}_{\mu \nu \alpha \beta} \bar{C}^{\mu \nu \alpha \beta} .
$$

The constant term in the r.h.s. of (114) is obviously irrelevant for the anomaly and the dependence on the conformal factor $\sigma$ may come only from

$$
\ln \left(-\frac{\square}{4 \pi \mu^{2}}\right)=2 \sigma+\ln \left(-\frac{\bar{\nabla}^{2}}{4 \pi \mu^{2}}\right)+\mathcal{O}\left(\sigma^{2}\right) .
$$

Obviously, this gives the standard expression for the corresponding term in the anomaly after we apply the prescription (61).

The same approach can be applied to the massless but conformal non-invariant limit $m^{2}=$ $0, \xi \neq 1 / 6$ in the $\int R\left(k_{R}\right) R$ term 6

$$
\begin{aligned}
& \left.\int d^{4} x \sqrt{-g} R\left\{\frac{1}{2 \epsilon}\left(\xi-\frac{1}{6}\right)^{2}+k_{R}(a)\right\} R\right|_{m \rightarrow 0}= \\
= & \int d^{4} x \sqrt{-g} R\left\{\frac{1}{2}\left[\frac{2}{4-n}-\ln \left(-\frac{\square}{4 \pi \mu^{2}}\right)\right]\left(\xi-\frac{1}{6}\right)^{2}+\frac{1}{12 \cdot 180(4 \pi)^{2}}\right\} R .
\end{aligned}
$$

The last term here is nothing but (113). It is remarkable that any deviation of $\xi$ from the precisely conformal value $1 / 6$ leads to the nonlocalities in the $\int R\left(k_{R}\right) R$-sector. If we keep in mind that the same also happens for any nonzero value of the mass, it becomes clear that the ambiguity of the $\square R$ term in the anomaly or, equivalently, of the $\int R^{2}$-term in the effective action is something quite subtle.

\footnotetext{
${ }^{6} \mathrm{~A}$ possible cosmological implications of this action have been considered in 51 .
} 


\subsubsection{Discontinuity of quantum corrections in the Proca theory}

The next interesting issue is the massless limit for the quantum corrections in the theory with softly broken gauge symmetry. Two most important examples of such theories are photon and graviton with tiny masses. We shall follow the recent paper [30] but consider only the massive vector theory.

Our purpose is to compare quantum contributions of massless and massive photons to the vacuum effective action in curved space-time. Consider the Proca model described by the action

$$
S_{P}=\int d^{4} x \sqrt{-g}\left\{-\frac{1}{4} F_{\mu \nu}^{2}+\frac{1}{2} M^{2} A_{\mu}^{2}\right\}
$$

The standard approach [15] gives the following expression for the one-loop contribution:

$$
\bar{\Gamma}^{(1)}=\frac{i}{2} \operatorname{Tr} \ln \left(\delta_{\alpha}^{\nu} \square-R_{\alpha}^{\mu}-M^{2} \delta_{\alpha}^{\mu}\right)-\frac{i}{2} \operatorname{Tr} \ln \left(\square-M^{2}\right) .
$$

In the massless limit the last term is twice the Faddeev-Popov ghost contribution, that is why we meet a discontinuity in this limit. An extra term is nothing but the contribution from some scalar field. In order to better understand the origin of this scalar, one can apply the Stückelberg procedure [117] and consider a new action

$$
S_{P}^{\prime}=\int d^{4} x \sqrt{-g}\left\{-\frac{1}{4} F_{\mu \nu}^{2}-\frac{1}{2} M^{2}\left(A_{\mu}-\frac{1}{M} \partial_{\mu} \varphi\right)^{2}\right\}
$$

This expression possesses invariance under the gauge transformation

$$
A_{\mu} \rightarrow A_{\mu}^{\prime}=A_{\mu}+\partial_{\mu} \xi \quad \text { and } \quad \varphi \rightarrow \varphi^{\prime}=\varphi+\xi M
$$

In the special gauge $\varphi=0$ we come back to the action (116). Concerning the quantum corrections, the gauge fixing dependence is irrelevant for the free fields actions such as (116) and (118). Hence, it is legitimate to use the most useful gauge fixing to evaluate the quantum contributions.

Using the linear gauge fixing condition $\chi=\nabla_{\mu} A^{\mu}-M \varphi$, the sum of the action (118) and the gauge fixing term, $S_{g f}=-\frac{1}{2} \int d^{4} x \sqrt{-g} \chi^{2}$, is cast into the form

$$
S^{\prime}=\frac{1}{2} \int d^{4} x \sqrt{-g}\left\{A^{\alpha}\left(\delta_{\alpha}^{\nu} \square-R_{\alpha}^{\mu}-M^{2} \delta_{\alpha}^{\mu}\right) A_{\nu}+\varphi\left(\square-M^{2}\right) \varphi\right\} .
$$

The Faddeev-Popov ghost's operator has the form $\hat{H}_{g h}=\square-M^{2}$, therefore the effective action is

$$
\begin{aligned}
\bar{\Gamma}^{(1)} & =\frac{i}{2} \operatorname{Tr} \ln \left(\delta_{\alpha}^{\nu} \square-R_{\alpha}^{\mu}-M^{2} \delta_{\alpha}^{\mu}\right) \\
& +\frac{i}{2} \operatorname{Tr} \ln \left(\square-M^{2}\right)-i \operatorname{Tr} \ln \left(\square-M^{2}\right),
\end{aligned}
$$

that is nothing but (117). An extra scalar was "hidden" in the massive term of the vector.

In order to understand the physical sense of the discontinuity of the massless limit, let us recall the result for the Proca field obtained in [61,

$$
\begin{aligned}
\bar{\Gamma}_{\text {vector }}^{(1)} & =\frac{1}{2(4 \pi)^{2}} \int d^{4} x \sqrt{-g}\left\{\frac{3}{2} M^{4} \cdot\left(\frac{1}{\epsilon}+\frac{3}{2}\right)+\frac{M^{2}}{2} R\left(\frac{1}{\epsilon}+1\right)\right. \\
& \left.+\frac{1}{2} C_{\mu \nu \alpha \beta}\left[\frac{13}{60 \epsilon}+k_{W}^{v}(a)\right] C^{\mu \nu \alpha \beta}+R\left[\frac{1}{72 \epsilon}+k_{R}^{v}(a)\right] R\right\} .
\end{aligned}
$$


This expression is quite similar to the one for the scalar field (110).

The most interesting for us is the nonlocal finite formfactor

$$
k_{W}^{v}(a)=-\frac{91}{450}+\frac{2}{15 a^{2}}-\frac{8 A}{3 a^{2}}+A+\frac{8 A}{5 a^{4}} .
$$

In the limit $M \rightarrow 0$ we obtain

$$
\frac{13}{60 \epsilon}+k_{W}^{v}(a) \rightarrow \frac{13}{60}\left(\frac{2}{4-n}-\gamma-\ln \frac{\square}{4 \pi \mu^{2}}\right)-\frac{38}{225}
$$

The divergence and finite constant terms can be canceled by local counterterm. The most relevant is the nonlocal term with $-\frac{13}{60} \ln \left(\square / 4 \pi \mu^{2}\right)$, which is a quantum contribution to the gravitational wave equation for the massless limit of the Proca model. The corresponding term derived for the gauge vector field is just $-\frac{1}{5} \ln \left(\square / 4 \pi \mu^{2}\right)$. The difference between the two coefficients $1 / 60=13 / 60-1 / 5$ is the contribution of an extra scalar field which was discussed above. In the massless limit this field still gives contribution to the vacuum effective action, leading to the discontinuity effect.

Qualitatively similar situation takes place for the massive spin-2 field contributions [47]. However, since such field can be formulated only on special backgrounds, there is no real possibility to meet the discontinuity in the essential non-local sector.

\section{Renormalization group for the cosmological constant}

Perhaps the reader already noticed that the expression for the effective action of vacuum (110) does not include nonlocal formfactors for the Einstein-Hilbert and cosmological terms. As a result we get zero $\beta$-functions for the corresponding parameters $\beta_{\Lambda}=\beta_{1 / G}=0$. The reason is that, in the perturbative in $h_{\mu \nu}$ approach, we can observe the decoupling for the parameters of the higher derivative terms, but not for the cosmological and inverse Newton constant. In other words, the physical predictions of the $\overline{\mathrm{MS}}$ renormalization scheme and the ones of the momentum subtraction renormalization scheme diverge completely at this point. Let us try to explain why this difference takes place.

\subsection{Once again on the physical renormalization group in curved space}

We keep in mind that the renormalization group running corresponds, in the UV limit, to the insertion of $\ln \left(\square / \mu^{2}\right)$-like formfactors into the effective action. However, as we already discussed in the previous sections, such insertions are not possible for the CC and $1 / G$.

Is it true that physical $\beta_{\Lambda}$ and $\beta_{1 / G}$ are equal to zero? It is clear that we have no sufficient data to claim this. The point is that the corresponding $\beta$-functions come from the contributions

of massive quantum fields. The consistent formulation of such fields on curved background require introducing the cosmological term into classical action. Then, the $g_{\mu \nu}=\eta_{\mu \nu}+h_{\mu \nu}$ approach implies an expansion over the flat background which is not a solution of the classical equations of motion. The only conclusion we have to make is that the momentum subtraction scheme and the flat-background expansion are not appropriate instruments for investigating the renormalization group for the cosmological constant. Some other methods should be developed before we can arrive at some formal results in this area. 
The natural option in this situation is to consider the calculations on some special backgrounds. For instance, the existing methods (e.g., $\zeta$-regularization) applicable on the de Sitter or anti de Sitter spaces [50], are very powerful in sense one can, sometimes, calculate the whole effective action. Unfortunately, the information obtained in this way is restricted because the curvature is constant and all derivatives acting on the curvature are zeros. The main disadvantage of the calculations on such backgrounds is that one can not see the non-localities of the EA. Furthermore it is not possible to distinguish between distinct higher derivative terms from one side and the Einstein-Hilbert and cosmological terms from other side.

\subsection{Running cosmological constant}

Since the possible running of the cosmological constant is an interesting and "hot" issue, one can approach it from the phenomenological side and consider applications to the "late" cosmology. As we have already explained in section 2, we shall associate the cosmic scale parameter with the Hubble parameter, $\mu_{c} \sim H$ [11, 110]. Let us notice that this identification of the scale has certain advantages over other possible choices [102].

In order to define the possible form of the scale-dependence of the quantum corrections to the vacuum energy we can recall the considerations presented in subsection 3.1 which were based on covariance. The quantum corrections can not be proportional to the odd powers of the Hubble parameter. Due to the smallness of $H_{0} \approx 10^{-42} \mathrm{GeV}$, compared to the critical density $\rho_{c}=\frac{3}{8 \pi} H_{0}^{2} M_{P}^{2} \approx 10^{-47} \mathrm{GeV}^{4}$, we need to consider only zero-order and second-order in $H$ corrections.

The zero-order corrections from the particle of the mass $m$ would be proportional to $m^{4}$. It is remarkable that the probable value of the neutrino mass $m_{\nu} \propto 10^{-12} \mathrm{GeV}$ gives the "quantum correction" to the energy density of vacuum $\delta \rho_{\Lambda} \sim m_{\nu}^{4}$ of the right order of magnitude [109]. The cosmological model based on this form of correction has been considered in [18]. At the same time the hypothesis $\delta \rho_{\Lambda} \sim m_{\nu}^{4}$ has an obvious weak point. If the neutrino gives quadratic contribution to $\rho_{\Lambda}$, why would not other fermions or bosons give such contributions too? There is nothing special in the neutrino's vacuum diagrams that could support the idea of neutrino's exclusive role here. Now, if we assume that other massive particles give quadratic contribution, the size of such contribution would be huge and destroy any reasonable cosmological model.

As far as the idea of non-decoupling meets serious obstacles, let us assume that the Appelquist \& Carazzone-like quadratic decoupling holds for the vacuum energy. In this case the contribution of the particle of a mass $m$ has the form $m^{2} \mu_{c}^{2}$, where $\mu_{c} \propto H$ is the Hubble parameter. It is important to keep in mind that the difference between the magnitude of Hubble parameter and the one of the masses of all known particles is huge. It is so huge that our intuition concerning the smallness of neutrino mass lies here! Let us see. We have $m / H_{0}$ is $10^{-30}$ for the typical neutrino mass, while in the QCD case we have $\Lambda_{Q C D} / H_{0} \sim 10^{-40}$ and for the Planck mass we have $M_{P} / H_{0} \sim 10^{-60}$. Hence, qualitatively the gap between the cosmic scale $\mu_{c}$ and neutrino is no greater than the one between the cosmic scale $\mu_{c}$ and the typical QCD scale or between the cosmic scale $\mu_{c}$ and the Planck scale. If we admit the potential importance of the neutrino loop or the nonperturbative vacuum effects of QCD at the cosmic scale, we have to admit that all existing particles must produce relevant contributions. And these contributions must be suppressed quadratically due to the covariance requirement. 
After all, our considerations lead to the following form of quantum correction to the vacuum energy density:

$$
\delta \rho_{\Lambda} \sim \sum_{i} S_{i} m_{i}^{2} H^{2}
$$

where the sum is over all massive particles, from neutrino up to the possible GUT constituents and beyond, to the hypothetical Planck-scale particles. The coefficient $S_{i}$ may be different for different particles and, in particular, one can expect it to have opposite signs for fermions and bosons. After this algebraic summation and adding the constant term (required by renormalizability), we arrive at the quite general expression for the vacuum energy density

$$
\rho_{\Lambda}(H)=\rho_{\Lambda}^{0}+\frac{3 \nu}{8 \pi} M_{P}^{2}\left(H^{2}-H_{0}^{2}\right)
$$

Here $\rho_{\Lambda}^{0}$ is the vacuum energy density in the present-day universe, $\rho_{\Lambda}(H)$ is the vacuum energy density and $\nu$ is the unique indefinite component of our model. The magnitude and sign of $\nu$ depends, according to the eq. (123), on the particle's spectrum and, mainly, on the spectrum of heaviest particles. Let us notice that the formula (124) holds even if we take the higher loop effects into account and even if there are some strong nonperturbative effects in the unknown high energy part of the particles spectrum. In this case the analog of the expression (123) will be, of course, more complicated due to the possible mass mixing, it will depend on the couplings, but the form of (124) will hold, because it is based only on covariance.

In fact, there is no certainty that the quadratic decoupling really takes place. What we can claim is that, if there is no cosmic scalar field (or some its substitute, like Chaplygin gas etc), the eq. (124) is the unique possible form of the non-constant vacuum energy. That is why this form of dependence is worthwhile to be explored in details. Imagine we detect someday the nonconstant vacuum energy. Then, in order to conclude that this indicates some new fundamental physics (it may be extra dimensions, quintessence etc) one has to rule out the corrections from the quantum matter fields on curved background. And these corrections, in the case of the vacuum energy, have the form (124).

It is easy to see that if there are no particles beyond the Minimal Standard Model, then the product $\nu M_{P}^{2}$ is about $M_{F}^{2} \sim 10^{5} \mathrm{GeV}^{2}$. In this case the magnitude of the second term in the r.h.s. of eq. (124) is about $10^{-80} \mathrm{GeV}^{4}$ in the recent universe with $H \approx H_{0}$, while $\rho_{\Lambda}^{0} \approx 10^{-42} \mathrm{GeV}^{4}$. Obviously, this means the quantum contributions are irrelevant and the cosmological constant is really a constant.

On the other hand, if there are particles with the mass close to $M_{P}$, then $\nu$ is of the order one and the quantum contribution is of the same order of magnitude as $\rho_{\Lambda}^{0}$. The standard assumption of the gap in the mass spectrum between the GUT scale $M_{X} \sim 10^{16} \mathrm{GeV}$ and $M_{P}$ produces the value of $\nu$ about $10^{-6}$. Of course, the presence of such particles does not automatically mean that the value of $\nu$ can not be much smaller, because the quantum contributions may cancel, e.g., due to supersymmetry.

The cosmological models based on the law (124) have been constructed in [112] and [111]. In both kinds of models the cosmological constant terms change together with the Hubble parameter. But if the vacuum energy density changes with time, this energy has to go somewhere! The difference between these two models constructed in [112] and [111] is the form of the conservation 
law. In the first case we assume the possibility of the energy exchange between the vacuum and matter sectors. In the second case such energy change is forbidden and the conservation law is provided due to the $H$-dependence of the Newton constant $G$.

The cosmological model of the first sort admits analytic solution for the conformal factor [112. The analysis of density perturbations has been performed in the framework of analog model [87] and also through the direct calculus [71]. In both cases the obtained limit $|\nu| \leq 10^{-6}$ is much stronger than the one which can be expected from SNAP [112. This bound corresponds to the GUT-like particle spectrum.

The model of the second kind [111] is a bit more complicated and the density perturbations have not been elaborated yet. However, since at small $\nu$ the two models behave quite similar, one can expect that the restrictions for $\nu$ will be also similar. It is interesting that this model predicts also a logarithmic scale dependence of the Newton constant

$$
G(H ; \nu)=\frac{G_{0}}{1+\nu \log \left(H^{2} / H_{0}^{2}\right)},
$$

where $G\left(H_{0}\right)=G_{0}=1 / M_{P}$. On the other hand, the universality of the effective action of vacuum means that, in general, there is similar dependence with $H$ traded for the general scale parameter $\mu$. In particular, in the astrophysical setting this means

$$
G(r)=\frac{G_{0}}{1+\nu \log \left(r_{0}^{2} / r^{2}\right)},
$$

where $r$ is the distance from the center of the galaxy. Then the "GUT-inspired" value $\nu \sim 10^{-6}$ is close to the one required for the approximately flat rotation curve in the spiral galaxies [111. So, the quantum corrections may be relevant also for the Dark Matter problem, affecting the gravitational law at the galactic scale. This distance-dependence does not affect the rotation curves inside the Solar System and represents a reasonable alternative to other models of modified gravitational law [80, 19].

The models developed in [112] and [111] show that the time-dependent vacuum energy and also distance-depending Newton constant can result from the semiclassical theory. However, the existence of the quadratic decoupling is hypothetical and needs, at the first place, a serious theoretical investigation. It would be extremely interesting to have a consistent QFT model for the effective action behind the possible CC running. In other words, we need a method for deriving the remnant non-local structures in the effective action of a massive fields. So far, we have no such model. One can prove that the effective action responsible for the CC running can not be of a finite polynomial order in curvatures [112, but this does not constitute the proof of no-running.

\section{Do we need Quantum Gravity?}

We have started our review from the discussion of Planck units, indicating the need of some kind of quantum gravity. However, formulating the physical theory requires defining the possible area of its application. In case of quantum gravity the first choice is the region of space-time where the typical energies are comparable to the Planck mass. Indeed, this can be achieved only in the vicinity of a singularity. 
The singularity is the natural feature of General Relativity [73, however it is well known that the Hawking-Penrose theorem can be violated by the semiclassical corrections coming from the quantum matter fields on classical metric background [55]. More concrete study has been performed in [3] for the case of cosmological singularity. The theory considered in [3] was based on the conformal anomaly, which we discussed in section 4 . The result is that the quantum corrections which lead to the Starobinsky inflation, can erase the initial Big Bang singularity. One can call this effect the back reaction of quantum matter on the vacuum. Despite the metric is not quantized, the form of the vacuum action gets modified due to the quantum corrections from matter loops and, as a result, in the new theory there is no singularity. Similar phenomenon can take place in the black hole case. According to the results of [58], already the higher derivative terms in the classical action of vacuum may switch off the singularity in the gravitational collapse. One can expect similar effect for the case of anomaly-induced effective action, in particular because the metric in the interior of the black hole is of the FRW-type [56]. The situation is expected to be same as in the cosmological case [3].

Finally, let us remark that the Schwarzschild solution is an essential idealization of the realistic situation when the black hole has a nonzero angular momentum. The quantum consideration, including the classification of the vacuum states of the black hole [12], which has been presented in the subsection 5.1, is very difficult to generalize for the rotating black hole case. Concerning the impact of quantum effects on the $r=0$ singularity, the geometric structure of the interior of the rotating black hole is much more complex than the one in a simplified Schwarzschild version. In particular, for the case of rotating black hole one meets a phenomenon which was called "mass inflation" 99]. The description of the possible global structure inside the rotating black hole has been given in [57] (see also [56]).

Generally speaking, the results of the existing studies of singularities in the theories with semiclassical corrections are not really conclusive. In particular, the presence of singularities may be affected by angular momentum or the choice of initial data in the case of the gravitational collapse and, definitely, by the uncertainty which we meet in the form of the quantum corrections. However, the possibility of a non-singular behavior can not be underestimated. If the disappearance of singularities in the physically relevant solutions is true, the Planck scale physics may become unobservable. For instance, as we already mentioned in the Introduction, the standard argument in favor of the quantization of the metric is an inconsistency of the semiclassical Einstein equations

$$
R_{\mu \nu}-\frac{1}{2} R g_{\mu \nu}=8 \pi G T_{\mu \nu}
$$

The l.h.s. of this equation is classical and deterministic while the r.h.s. depends on quantum variables and therefore oscillates. However, there is a Planck suppression of such oscillations, hence they should be irrelevant at the energies much smaller than the Planck ones. Then the situation becomes dramatically different if the access to the Planck energy is really screened by the semiclassical modifications of geometry.

There is an alternative possibility. One may hope to observe the effects of Quantum Gravity or, e.g., string theory as small effects at low energies. However, similar small effects may be, in principle, produced in the framework of the semiclassical theory.

For example, the potentially observable time-dependent Dark Energy in the universe may be an effect of quintessence or Chaplygin gas, that would mean a qualitatively new physics, 
originating from the string theory. Similar effect can be also produced by quantum gravity if we accept the popular hypothesis on the non-Gaussian fixed point in this theory [101]. However, quite similar effect of a scale-dependent vacuum energy may come also from the semiclassical gravity [112, 111]. The important difference is that, as we stressed in the Introduction, the quantum effect of matter fields on curved background is something which certainly exists, even if we do not know well how to evaluate it. On the contrary, we are not certain that there is some physical reality behind the string theory or quantum gravity. Therefore, the reliable evaluation of the semiclassical contributions is a necessary step in the study of physical significance of quantum gravity or string theory. Before these contributions are sufficiently studied, one can not draw conclusions about a qualitatively new physics.

\section{Conclusions}

The development of experimental and observational technologies produce an increasing amount and quality of the information about the Universe. For a while, the existing data fit with the $\Lambda \mathrm{CDM}$ model, may be with some small modifications like introducing certain warmness of the Dark Matter. However, in the future one can expect to have an access to a more detailed description of the expansion of the Universe. It can not be ruled out that, after all, the Dark Energy will be variable [103]. This possibility constitutes a strong challenge for the theory which has to prepare reasonable explanations of this possible variation.

Does it mean the qualitatively new physics which manifests itself in the form of some cosmic field or cosmic-scale matter? Such matter would be something really unusual, because it must have a very small (if nonzero) mass and, at the same time, provide self-interaction on the cosmic distances. Since nothing like that have been ever seen in the laboratories, such cosmic entities would definitely mean that we met something beyond the Standard Model or its conventional extensions. At the same time, one can not rule out that the variable Dark Energy is just the result of the quantum effects of matter on the classical metric background. Similar situation takes place in the study of inflation. The presence of inflaton can signal some qualitatively new physics. However the inflation may be just caused by the Higgs field of the Standard Model with the nonminimal coupling, as it was suggested in [24], or result from the quantum corrections to the classical vacuum action of vacuum in the framework of the Starobinsky model [114], or its modified version [95]. The difference is that the vacuum quantum effects or the nonminimally coupled Higgs are nothing but the manifestations of the existing physics. According to the conventional scientific logic, these options must "go first" and only if they fail to explain a new phenomena, introducing qualitatively new objects must be seriously considered.

The main obstacle in performing this program is that we have no tools to check, for example, whether the vacuum quantum effects are really driving the Dark Energy. This happens because the relevant part of the vacuum effective action comes from the massive fields, it is non-local and very complicated. In this review we have described the present-day situation with quantum corrections. As we have seen, the situation is rather optimistic for the massless conformal fields. Furthermore, we have some models (more or less reliable) for the very light quantum fields and, to some extent, for the very heavy quantum fields. Unfortunately, we have no model for the intermediate case. 
The QFT in curved space-time is about 50 years old. At the moment we have a very good understanding of the renormalization in curved space and of the related issues like trace anomaly. Our main hope is to achieve better knowledge of the relevant quantum effects in the next decades and, in this sense, to match the achievements of observational cosmology, experimental high energy physics and phenomenology.

Acknowledgments. Author is very grateful to M. Asorey, R. Balbinot, I.L. Buchbinder, A. Fabbri, J. Fabris, E. Gorbar, G. de Berredo-Peixoto, A. Pelinson and J. Solà for numerous discussions and common works and to many other colleagues, especially to A.A. Starobinsky and V.P. Frolov, for clarifying conversations and correspondence. The present review is partially based on the lectures which were given at the Universidad de Zaragoza (1995), Centro Brasileiro de Pesquisas Físicas (1996), Universitat Autònoma de Barcelona (1997), Universidade Federal de Juiz de Fora (1997 and 2007) and at the summer schools "Quantum Summer" at the Universidade Federal do Espírito Santo (1998 and 2007). I am really thankful to the organizers of these courses and to those who were attending lectures and asked questions. The work has been partially supported by the PRONEX project and research grants from FAPEMIG (MG, Brazil) and CNPq (Brazil), PRONEX project from FAPES (ES, Brazil) and CNPq and by the individual grants from $\mathrm{CNPq}$ and ICTP.

\section{References}

[1] S.L. Adler, Rev. Mod. Phys. 54 (1982) 729.

[2] J.D. Anderson, Ph.A. Laing, E.L. Lau, A.S. Liu, M. Martin Nieto and S.G. Turyshev, Phys. Rev. D65 082004 (2002).

[3] P. Anderson, Phys. Rev. D28 (1983) 271; D29 (1984) 615; D29 (1986) 1567.

[4] P.R. Anderson, E. Mottola, R. Vaulin, Stress Tensor from the Trace Anomaly in ReissnerNordstrom Spacetimes. Phys.Rev. D76 (2007) 124028; gr-qc/0707.3751.

[5] I. Antoniadis and E.T. Tomboulis, Phys. Rev. D33 (1986) 2756.

[6] M. Asorey, E.V. Gorbar and I.L. Shapiro, Class. Quant. Grav. 21 (2003) 163.

[7] M. Asorey, E.V. Gorbar and I.L. Shapiro, Class. Quant. Grav. 21 (2003) 163;

M. Asorey, G. de Berredo-Peixoto and I.L. Shapiro, Phys. Rev. D74 (2006) 124011.

[8] M. Asorey, J.L. López and I.L. Shapiro, Int. Journ. Mod. Phys. A12 (1997) 5711.

[9] T. Appelquist and J. Carazzone, Phys. Rev. 11D (1975) 2856.

[10] I.G. Avramidi, Covariant methods for the calculation of the effective action in quantum field theory and investigation of higher-derivative quantum gravity, (Ph.D. thesis at Moscow State University, 1986), hep-th/9510140, Heat Kernel and Quantum Gravity, (Springer-Verlag, 2000). 
[11] A. Babic, B. Guberina, R. Horvat, H. Štefančić, Phys. Rev. D71 (2005) 124041.

[12] R. Balbinot, A. Fabbri and I.L. Shapiro, Phys. Rev. Lett. 83 (1999) 1494; Nucl. Phys. B559 (1999) 301.

[13] R. Balbinot and A. Fabbri, Phys. Rev. D59 (1999) 044031;

F.C. Lombardo, F.D. Mazzitelli and J.G. Russo, Phys. Rev. D59 (1999) 064007.

[14] A.O. Barvinsky, Phys. Lett. B572 (2003) 109.

[15] A.O. Barvinsky and G.A. Vilkovisky, Phys. Rep. 119, (1985) 1.

[16] A.O. Barvinsky and G.A. Vilkovisky, Nucl. Phys. 333B (1990) 471;

I. G. Avramidi, Yad. Fiz. (Sov. Journ. Nucl. Phys.) 49 (1989) 1185.

[17] A.O. Barvinsky, Yu.V. Gusev, G.A. Vilkovisky and V.V. Zhitnikov, The One loop effective action and trace anomaly in four-dimensions, Nucl.Phys. B439 (1995) 561 [hep-th/9404187].

[18] F. Bauer, Class. Quant. Grav. 22 (2005) 3533.

[19] J.D. Bekenstein, Phys. Rev. D70 (2004) 083509; Erratum-ibid. D71 (2005) 069901.

[20] G. de Berredo-Peixoto, E.V. Gorbar and I.L. Shapiro, Class. Quant. Grav. 21 (2004) 2281.

[21] G. de Berredo-Peixoto and I.L. Shapiro, Phys. Lett. 514B (2001) 377.

[22] O. Bertolami and J. Páramos, Class. Quantum Gravity 21 (2004) 3309;

[23] O. Bertolami, C. Boehmer, T. Harko and F.S.N. Lobo, Phys. Rev. D75 (2007) 104016.

[24] F. Bezrukov, M. Shaposhnikov, The Standard Model Higgs boson as the inflaton. hepth/0710.3755.

[25] N.D. Birell and P.C.W. Davies, Quantum Fields in Curved Space (Cambridge University Press, Cambridge, 1982).

[26] D.G. Boulware, Phys. Rev. D11 (1975), 1404.

[27] L.S. Brown and J.P. Cassidy, Phys. Rev. D15 (1977) 2810.

[28] I.L. Buchbinder and S.D. Odintsov, Lett. Nuovo Cim. 42 (1985) 379.

[29] I.L. Buchbinder, I.L. Shapiro and E.G. Yagunov, Mod. Phys. Lett. A5 (1990) 1599.

[30] I.L. Buchbinder, G. de Berredo-Peixoto, I.L. Shapiro, Phys. Lett. B649 (2007) 454.

[31] I.L. Buchbinder, S.D. Odintsov and I.L. Shapiro, Effective Action in Quantum Gravity (IOP Publishing, Bristol, 1992).

[32] I.L. Buchbinder, Theor. Math. Phys. 61 (1984) 393.

[33] I.L. Buchbinder and J.J. Wolfengaut, Class. Quant. Grav. 5 (1988) 1127; 
[34] T. S. Bunch and L. Parker, Phys. Rev. D20 (1979) 2499;

[35] I.L. Buchbinder, S.D. Odintsov and I.L. Shapiro, Phys. Lett. B162 (1985) 92;

[36] R. Camporesi, Phys. Rept. 196 (1990) 1.

[37] P. Candelas, Phys. Rev. D21 (1980) 2185;

D.N. Page, Phys. Rev. D25 (1982) 1499;

M.R. Brown, A.C. Ottewill and D.N. Page, Phys. Rev. D33 (1986) 2840;

V.P. Frolov and A.I. Zelnikov, Phys. Rev. D35 (1987) 3031;

P.R. Anderson, W.A. Hiscock and D.A. Samuel, Phys. Rev. D51 (1995) 4337.

[38] D.F. Carneiro, E.A. Freitas, B. Gonçalves, A.G. de Lima and I.L. Shapiro, Grav. and Cosm. 40 (2004) 305; gr-qc/0412113.

[39] T.P. Cheng, E. Eichten and L.-F. Li, Phys. Rev. D9 (1974) 2259.

[40] S.M. Christensen, Phys. Rev. D14 (1976) 2490; D17 (1978) 946.

[41] S.M. Christensen and S.A. Fulling, Phys. Rev. D15 (1977) 2088.

[42] S. Deser, Ann.Phys. 59 (1970) 248.

[43] S. Deser and P. van Nieuwenhuisen, One-loop divergences of quantized Einstein-Maxwell fields. Phys. Rev. 10D, (1974) 401-410.

[44] S. Deser and B. Tekin, Phys. Rev. D67 (2003) 084009.

[45] A. Dobado, A.L. Maroto, Phys. Rev. D60 (1999) 104045.

[46] S. Dodelson, Modern Cosmology (Academic Press, New York, 2003).

[47] F.A. Dilkes, M.J. Duff, J.K. Liu and H. Sati, Phys. Rev. Lett. 87 (2001) 041301;

M.J. Duff, J.T. Liu and H. Sati, Phys. Lett. B516 (2001) 156.

[48] M.J. Duff, Class. Quant. Grav. 11 (1994) 1387 hep-th/9308075.

[49] M.J. Duff, Nucl. Phys. B125 (1977) 334;

S. Deser, M.J. Duff and C. Isham, Nucl. Phys. B111 (1976) 45.

[50] E. Elizalde, Zeta regularization techniques with applications, World Scientific, 1994;

D.V. Vassilevich, Phys. Rept. 388 (2003) 279, see also further references therein.

[51] D. Espriu, T. Multamaki, E.C. Vagenas, Phys. Lett. B628 (2005) 197.

[52] J.C.Fabris, A.M.Pelinson and I.L.Shapiro, Nucl. Phys. B597 (2001) 539.

[53] M.V. Fischetti, J.B. Hartle and B.L. Hu, Phys. Rev. D20 (1979) 1757.

[54] E.S. Fradkin and A.A. Tseytlin, Phys. Rept. 119 (1985) 233. 
[55] L.H. Ford, Int. J. Theor. Phys. 42 (2003) 1219.

[56] V.P.Frolov and I.D.Novikov, Black Hole Physics - Basic Concepts and New Developments. (Kluwer Academic Publishers, 1989).

[57] V.P. Frolov, M.A. Markov, V.F. Mukhanov, Phys. Rev. D41 (1990) 383.

[58] V.P. Frolov, G.A. Vilkovisky, Phys. Lett. B106 (1981) 307;

[59] P.B. Gilkey, J. Diff. Geom. 10 (1975) 601.

[60] J. Gomis, S. Weinberg, Nucl. Phys. B469 (1996) 473.

[61] E.V. Gorbar, I.L. Shapiro, JHEP 02 (2003) 021; JHEP 06 (2003) 004.

[62] E.V. Gorbar and I.L. Shapiro, JHEP 02 (2004) 060.

[63] M.B. Green, J.H. Schwarz and E. Witten, Superstring Theory. (Cambridge University Press, Cambridge, 1987).

[64] A.A. Grib, S.G. Mamaev and V.M. Mostepanenko, Quantum Effects in Intensive External Fields. (Moscow, Atomizdat. In Russian, 1980).

[65] A.H. Guth, Phys. Rev. D23 (1981) 347.

[66] W. Israel, Phys. Lett. A57 (1976), 107; J.B. Hartle and S.W. Hawking, Phys. Rev. D13 (1976), 2188.

[67] Johnston D.A., Nucl.Phys. 297B (1988) 721.

[68] S.J. Hathrell, Annals Phys. 139 (1982) 136.

[69] S.W. Hawking, Comm. Math. Phys. 55 (1977) 133.

[70] S. W. Hawking, Nature (London) 248 (1974), 30; Commun. Math. Phys. 43 (1975), 199, Erratum-ibid. 46 (1976) 206.

[71] J.C. Fabris, I.L. Shapiro, J. Solà, JCAP 0702 (2007) 016.

[72] S.W. Hawking, T. Hertog and H.S. Real, Phys.Rev. D63 (2001) 083504.

[73] S.W. Hawking, R. Penrose, Proc. Roy. Soc. Lond. A314 (1970) 529.

[74] G. t'Hooft and M. Veltman, Ann. Inst. H. Poincare A20 (1974) 69.

[75] E. Kolb and M. Turner, The Very Early Universe (Addison-Wesley, New York, 1994).

[76] L.D. Landau and E.M. Lifshits, Field Theory (Pergamon Press, London).

[77] A. Linde, Phys. Lett. B108 (1982) 389; Particle Physics and Inflationary Cosmology (Harwood, Chur, Switzerland, 1990).

[78] A.L. Maroto and I.L. Shapiro, Phys.Lett. 414B (1997) 34. 
[79] P. O. Mazur and E. Mottola, Phys. Rev. D64 (2001) 104022.

[80] M. Milgrom, Astrophys. J. 270 (1983) 365; 371.

[81] V. Mukhanov, Physical Foundations of Cosmology (Cambridge University Press, 2005).

[82] V.F. Mukhanov and G.V. Chibisov, JETP Lett. 33 (1981) 532; JETP (1982) 258.

[83] V. Mukhanov and S. Winitzki, Introduction to Quantum Effects in Gravity (Cambridge University Press, 2007).

[84] V. Mukhanov, A. Wipf and A. Zelnikov, Phys. Lett. B332 (1994) 283.

[85] D.L. Nacir and F.D. Mazzitelli, Phys. Rev. D75 (2007) 024003.

[86] B.L. Nelson and P. Panangaden, Phys.Rev. D25 (1982) 1019.

[87] R. Opher and A. Pelinson, Braz. J. Phys. 35 (2005) 1206; Phys. Rev. D70 (2004) 063529;

G. Olivares, F. Atrio-Barandela and D. Pavon, Phys.Rev. D74 (2006) 043521.

[88] T. Padmanabhan, Structure Formation in the Universe (Cambridge University Press, 1993).

[89] L. Parker and S.A. Fulling, Phys. Rev. D9 (1974) 341;

S.A. Fulling, L. Parker and B.L. Hu, Phys. Rev. D10 (1974) 3905.

[90] L. Parker, D.J. Toms, Phys. Rev. D31 (1985) 2424;

I. Jack and L. Parker, Phys. Rev. D31 (1985) 2439.

[91] L. Parker, A. Raval, Phys. Rev. D60 (1999) 063512, Erratum-ibid. D67 (2003) 029901; Phys. Rev. D60 (1999) 123502, Erratum-ibid. D67 (2003) 029902;

L. Parker, D.A.T. Vanzella, Phys. Rev. D69 (2004) 104009.

[92] L. Parker and D.J. Toms, Phys. Rev. 29D (1984) 1584.

[93] R.D. Peccei, J. Solà, C. Wetterich, Phys. Lett. 195B (1987) 183;

C. Wetterich, Nucl. Phys. B302 (1988) 668;

W. Buchmuller, N Dragon, Nucl. Phys. B321 (1989) 207;

J. Solà, Phys.Lett. B228 (1989) 317, Int. J. Mod. Phys. A5 (1990) 4225;

G.D. Coughlan, I. Kani, G.G. Ross, G. Segre, Nucl. Phys. B316 (1989) 469;

E.T. Tomboulis, Nucl. Phys. B329 (1990) 410.

[94] P. J. E. Peebles, Principles of Physical Cosmology. (Princeton University Press, Princeton, 1993).

[95] A.M. Pelinson, I.L. Shapiro and F.I. Takakura, Nucl. Phys. B648 (2003) 417.

[96] A.M. Pelinson, I.L. Shapiro and F.I. Takakura, Nucl. Phys. (Proc. Suppl.) B127 (2004) 182. 
[97] M.E. Peskin and D.V. Schroeder, An Introduction to Quantum Field Theory (AddisonWesley, NY, 1995).

[98] A.Z.Petrov, Einstein Spaces. (Pergamon, Oxford, 1969).

[99] E. Poisson, W. Israel, Phys.Rev. D41 (1990) 1796.

[100] R.J. Riegert, Phys. Lett. 134B (1980) 56;

E.S. Fradkin and A.A. Tseytlin, Phys. Lett. 134B (1980) 187.

[101] M. Reuter, Phys. Rev. D57 (1998) 971;

O. Lauscher and M. Reuter, Phys. Rev. D65 (2002) 025013.

[102] M. Reuter, C. Wetterich, Phys. Lett. B188 (1987) 38;

A. Bonanno and M. Reuter, Phys. Lett. B527 (2002) 9; Phys. Rev. D65 (2002) 043508.

[103] V. Sahni and A. Starobinsky, Int. J. Mod. Phys. 9 (2000) 373;

S.M. Carroll, Living Rev. Rel. 4 (2001) 1;

T. Padmanabhan, Phys. Rept. 380 (2003) 235;

J. Solà, Nucl. Phys. Proc. Suppl. 95 (2001) 29.

[104] A.D. Sakharov, Sov. Phys. Dokl. 12 (1968) 1040.

[105] R. Schutzhold, Phys. Rev. Lett. 89, 081302 (2002).

[106] I.L. Shapiro, PoS-JHEP 03 (2006) 1, hep-th/0610168].

[107] I. L. Shapiro and A. G. Jacksenaev, Phys. Lett. B324 (1994) 284.

[108] I.L. Shapiro, J. Solà, Phys. Lett. B530 (2002) 10;

I.L. Shapiro, Int. Journ. Mod. Phys. 11D (2002) 1159.

[109] I.L. Shapiro, J. Solà, Phys. Lett. 475B (2000) 236.

[110] I.L. Shapiro, J. Solà, JHEP 02 (2002) 006.

[111] I.L. Shapiro, J. Solà, H. Stefancic, JCAP 0501 (2005) 012.

[112] I.L. Shapiro, J. Solà, C. España-Bonet, P. Ruiz-Lapuente, Phys. Lett. 574B (2003) 149; JCAP 0402 (2004) 006;

I.L. Shapiro, J. Solà, Nucl. Phys. Proc. Suppl. 127 (2004) 71.

[113] J. Solà, Dark energy: A Quantum fossil from the inflationary Universe?. hep-th/0710.4151.

[114] A.A. Starobinski, Phys.Lett. 91B (1980) 99; Nonsingular Model of the Universe with the Quantum-Gravitational De Sitter Stage and its Observational Consequences, Proceedings of the second seminar "Quantum Gravity", pp. 58-72 (Moscow, 1982); JETP Lett. 30 (1979) 719; 34 (1981) 460; Let.Astr.Journ. (in Russian), 9 (1983) 579. 
[115] K.S. Stelle, Phys. Rev. D16, 953 (1977).

[116] K.S. Stelle, Gen. Rel. Grav. 9 (1978) 353.

[117] E.C.G. Stückelberg, Helv. Phys. Acta. 30 (1957) 209.

[118] E. Tomboulis, Phys. Lett. 70B (1977) 361.

[119] D.J. Toms, Phys. Lett. B126 (1983) 37;

L. Parker, D.J. Toms, Phys. Rev. D32 (1985) 1409.

[120] W.G. Unruh, Phys. Rev. D14 (1976), 870.

[121] R. Utiyama, B.S. DeWitt, J. Math. Phys. 3(1962) 608.

[122] A. Vilenkin, Phys. Rev. D32 (1985) 2511.

[123] G.A. Vilkovisky, Ann.Phys. 321(2006)2717; Phys.Lett. B638(2006)523; B634(2006)456.

[124] G.A. Vilkovisky, Lect. Notes Phys. 737 (2008) 729; arXiv:0712.3379.

[125] B.L. Voronov, I.V. Tyutin, Theor. Math. Phys. 50 (1982) 218; 52 (1982) 628;

B.L. Voronov, P.M. Lavrov, I.V. Tyutin, Yad. Fiz. 36 (1982) 498 (Engl. translation: Sov. J. Nucl. Phys. 36 (1982) 292);

P.M. Lavrov, I.V. Tyutin, Yad. Fiz. 41 (1985) 1658 (Engl. translation: Sov. J. Nucl. Phys. 41 (1985) 1049).

[126] R.M. Wald, General Relativity. (University of Chicago Press, 1984).

[127] S. Weinberg, Gravitation and Cosmology. (Wiley, New York, 1972).

[128] S. Weinberg, Rev. Mod. Phys., 61 (1989) 1.

[129] B.S. DeWitt, Dynamical Theory of Groups and Fields. (Gordon and Breach, 1965).

[130] B.S. DeWitt, Phys. Rep. C19 (1975) 297.

[131] S. Weinberg, The Quantum Theory of Fields: I. Foundations; II. Modern Applications. (Cambridge University Press, Cambridge, 1995).

[132] Ya.B. Zeldovich, 6 (1967) 883.

[133] B. Zwiebach, Phys. Lett. 156B (1985) 315;

S. Deser and A.N. Redlich, Phys. Lett. 176B (1986) 350;

A.A. Tseytlin, Phys. Lett. 176B (1986) 92.

[134] Ya.B. Zeldovich, A.A. Starobinsky, Zh. Eksp. Teor. Fiz.61 (1971) 2161. Eng. translation: Sov. Phys. JETP 34 (1972) 1159. 